Projets

de paysage

\section{Projets de paysage}

Revue scientifique sur la conception et l'aménagement de l'espace

$22 \mid 2020$

Forêt et paysage

\title{
Quelles trajectoires paysagères pour les massifs forestiers militarisés du Grand-Est?
}

Essai de typologie à partir de l'étude des forêts de Bitche, de Haguenau et du Bienwald-Mundat

The Evolving Landscapes of the Militarised Forest Massifs of the Grand-Est Region of France - An Attempt to Establish a Typology Based on a Study of the Bitche, Haguenau and Bienwald-Mundat Forest Massifs

Denis Mathis, Emmanuel Chiffre, Christelle Balouzat-Loubet, Tanguy Niederlander et Anne Mathis

\section{(2) OpenEdition}

Journals

Édition électronique

URL : http://journals.openedition.org/paysage/8862

DOI : 10.4000/paysage.8862

ISSN : 1969-6124

\section{Éditeur :}

École nationale supérieure du paysage de Versailles-Marseille, Institut national des sciences appliquées Centre Val de Loire - École de la nature et du paysage, École nationale supérieure d'architecture et de paysage de Bordeaux, École nationale supérieure d'architecture et de paysage de Lille, Agrocampus Angers

Référence électronique

Denis Mathis, Emmanuel Chiffre, Christelle Balouzat-Loubet, Tanguy Niederlander et Anne Mathis, "Quelles trajectoires paysagères pour les massifs forestiers militarisés du Grand-Est? », Projets de paysage [En ligne], 22 | 2020, mis en ligne le 21 juillet 2020, consulté le 24 juillet 2020. URL : http:// journals.openedition.org/paysage/8862 ; DOI : https://doi.org/10.4000/paysage.8862

Ce document a été généré automatiquement le 24 juillet 2020.

Projets de paysage 


\section{Quelles trajectoires paysagères pour les massifs forestiers militarisés du Grand-Est?}

Essai de typologie à partir de l'étude des forêts de Bitche, de Haguenau et du Bienwald-Mundat

The Evolving Landscapes of the Militarised Forest Massifs of the Grand-Est Region of France - An Attempt to Establish a Typology Based on a Study of the

Bitche, Haguenau and Bienwald-Mundat Forest Massifs

Denis Mathis, Emmanuel Chiffre, Christelle Balouzat-Loubet, Tanguy Niederlander et Anne Mathis

1 Les frontières de la région Grand-Est sont des territoires marqués par la militarisation, la surmilitarisation, voire l'hypermilitarisation ${ }^{1}$ dont l'empreinte a structuré tout autant les espaces urbains que ruraux. Cette militarisation a construit un paysage hybride, qui associe et surimpose aux paysages civils une multitude d'infrastructures (casernes, camps, routes, terrains de manœuvre, zones inondables, casemates, forts...). Ces empilements d'objets militaires organisent un territoire de défense. Cependant, ces derniers subissent le processus de la démilitarisation qui est le résultat de la professionnalisation des armées et de la redistribution des forces militaires à l'échelle $\mathrm{du}$ territoire national. Devenue un enjeu d'aménagement (Ginet, 2011), la démilitarisation, souvent apatrimoniale, a rétrocédé au domaine civil (public ou privé) des éléments constitutifs des systèmes de défense désormais neutralisés, des friches et des cicatrices des anciens aménagements militaires. Ce démantèlement a fragmenté les dispositifs de défense faisant perdre progressivement tout ou partie de la cohérence des paysages. Aussi l'empreinte militaire s'est estompée lentement en fonction des phases d'abandon et l'ensemble du dispositif tend à disparaître ou est oublié des mémoires. Comme pour les polémopaysages ${ }^{2}$, cet effacement a été important au sein des espaces ouverts, et c'est finalement au cœur des espaces fermés que les traces et vestiges de la militarisation sont les mieux préservés et fossilisés. Les massifs forestiers 
constituent des capsules où ces vestiges restent nombreux, témoins visuels de l'hypermilitarisation des frontières.

Par massif forestier nous retiendrons l'approche de Jean-Pierre Husson et Xavier Rochel (2009), qui le définissent comme « un ensemble foncier (une forêt domaniale, communale, sectionale ou privée) ou, dans une acception probablement plus pertinente en géographie, comme un ensemble boisé distinct, plus ou moins homogène, à l'échelle paysagère ou régionale ». La militarisation des massifs a construit un paysage qui incorpore à la forêt des éléments complexes, de formes et de tailles très diverses (châteaux forts, routes militaires, camps militaires, lignes défensives...). Leur abandon total ou partiel laisse apparaitre une friche militaire en voie de fossilisation. Or, ce paysage militarisé est plus facilement préservé sous le couvert forestier. En effet, la gestion forestière des massifs ne nécessite pas l'effacement de ces infrastructures déclassées par l'armée lors de la démilitarisation. Progressivement, elles ont été oubliées, laissées partiellement en jachère ou sanctuarisées, voire scénarisées et valorisées dans le cadre de la gestion actuelle des massifs. Afin d'envisager les trajectoires paysagères et territoriales des héritages paysagers de ces espaces militarisés, nous avons retenu trois massifs forestiers frontaliers (figure 1) :

- le massif forestier des Vosges du Nord et plus particulièrement le «Pays couvert » du Pays du Bitche (Moselle). C'est dans le cadre de l'Observatoire Hommes-Milieux (OHM), au sein du projet dédié à l'empreinte militaire du Pays de Bitche, que nous retenons l'observation de ce massif où l'empilement des vestiges (des châteaux forts à la ligne Maginot) constitue l'élément le plus marquant. Le cœur du massif est constitué par le camp militaire de Bitche (3 $285 \mathrm{ha}$ ) acteur majeur de sa préservation environnementale ;

- le massif forestier de Haguenau avec le déploiement de la ligne Maginot. Sans ouvrage majeur, le massif et plus particulièrement la forêt indivise font l'objet d'un inventaire des vestiges de la ligne Maginot dans une démarche de classement de label Forêt d'Exception ${ }^{3}$. La question de la valorisation et de la sécurisation des sites militaires donne lieu actuellement à une réflexion dans le cadre d'une gestion plus globale du massif ;

- le massif forestier du Bienwald-Mundat de part et d'autre de la frontière franco-allemande sur le cours de la Lauter. Le massif abrite les vestiges de la ligne de la Lauter ou de Wissembourg, ligne de fortifications de campagne de la guerre de Succession d'Espagne (1701-1714) et régulièrement réactivée durant les ${ }_{x v i i i}{ }^{e}$ et ${ }_{\text {xix }}{ }^{\mathrm{e}}$ siècles. La forêt abrite également du côté allemand les vestiges, en grande partie effacés, de la ligne Siegfried ou Westwall datant de la Seconde Guerre mondiale. 


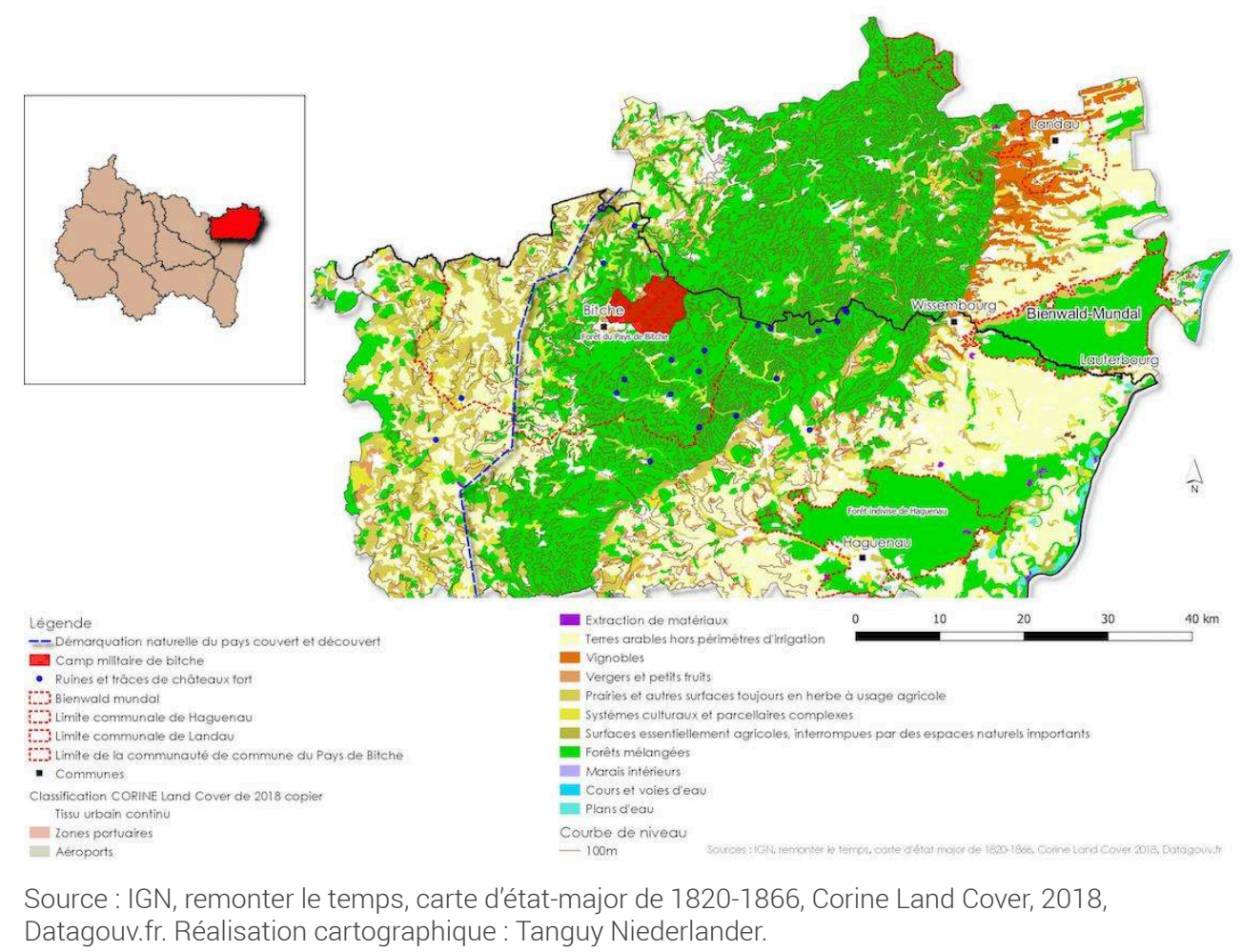

Ces trois massifs forestiers permettent de souligner tout à la fois la richesse et la diversité des formes de la militarisation, mais aussi la place de ces marqueurs paysagers dans la gestion territoriale de ces forêts associant ou non l'empreinte militaire.

\section{Des massifs forestiers frontaliers et militarisés entre les Vosges et le Rhin}

4 La militarisation des frontières du Grand-Est s'est faite en fonction de déploiements multiples et de la stabilisation des rapports de force. Si la cristallisation des frontières a permis de déployer, voire d'empiler, des infrastructures de défense, les phases de remise en cause de la frontière ont délaissé les éléments les moins réutilisables. Les massifs forestiers ont permis de les préserver par fossilisation, alors qu'au sein des espaces ouverts ces éléments ont eu tendance à être effacés.

\section{Le paysage militaire du massif forestier des Vosges du Nord}

5 Cette forte présence militaire marquée depuis le Moyen Âge par la cristallisation des éléments frontaliers entre seigneuries, puis entre France et Saint-Empire romain germanique, enfin entre France et Allemagne, a failli prendre fin avec la dissolution du $4^{\mathrm{e}}$ régiment de cuirassiers en 1997, puis du $57^{\mathrm{e}}$ régiment d'artillerie en 2009 . Ce départ définitif de l'armée a été remis en cause par l'arrivée du $16^{\mathrm{e}}$ bataillon de chasseurs en 2010. Le traumatisme de la démilitarisation est vu comme l'acte fondateur de l'Observatoire Hommes-Milieux (OHM) Pays de Bitche ${ }^{4}$. Le processus de militarisation s'est fait en trois temps. 


\section{Le temps des châteaux et des frontières}

6 La question des châteaux forts relève tout à la fois de la militarisation et de la démilitarisation du territoire. Zone de conflits et de tensions à l'époque médiévale, le Pays de Bitche est un territoire de passage avec de nombreux « conduits » routiers. Les châteaux assurent le marquage et le contrôle territorial de ces axes routiers. Le XVII siècle, avec l'irruption des armées françaises dans la région, est un temps de destruction de ces points d'appui militaires. Ces ruines, reconquises par la végétation, sont remises en scène par les associations du Club vosgien à partir de 1872 (figure 2). Seul le château de Bitche, après sa destruction, est converti en citadelle (1741-1754) par les travaux de Cormontaigne (figure 3). Son périmètre est étendu avec la construction $\mathrm{du}$ fort Saint-Sébastien (1846-1852) et la mise en œuvre de redoutes au sommet du Grand-Hohékirkel.

Figure 2. Les ruines du château du Falkenstein, vestiges de la militarisation des anciennes frontières

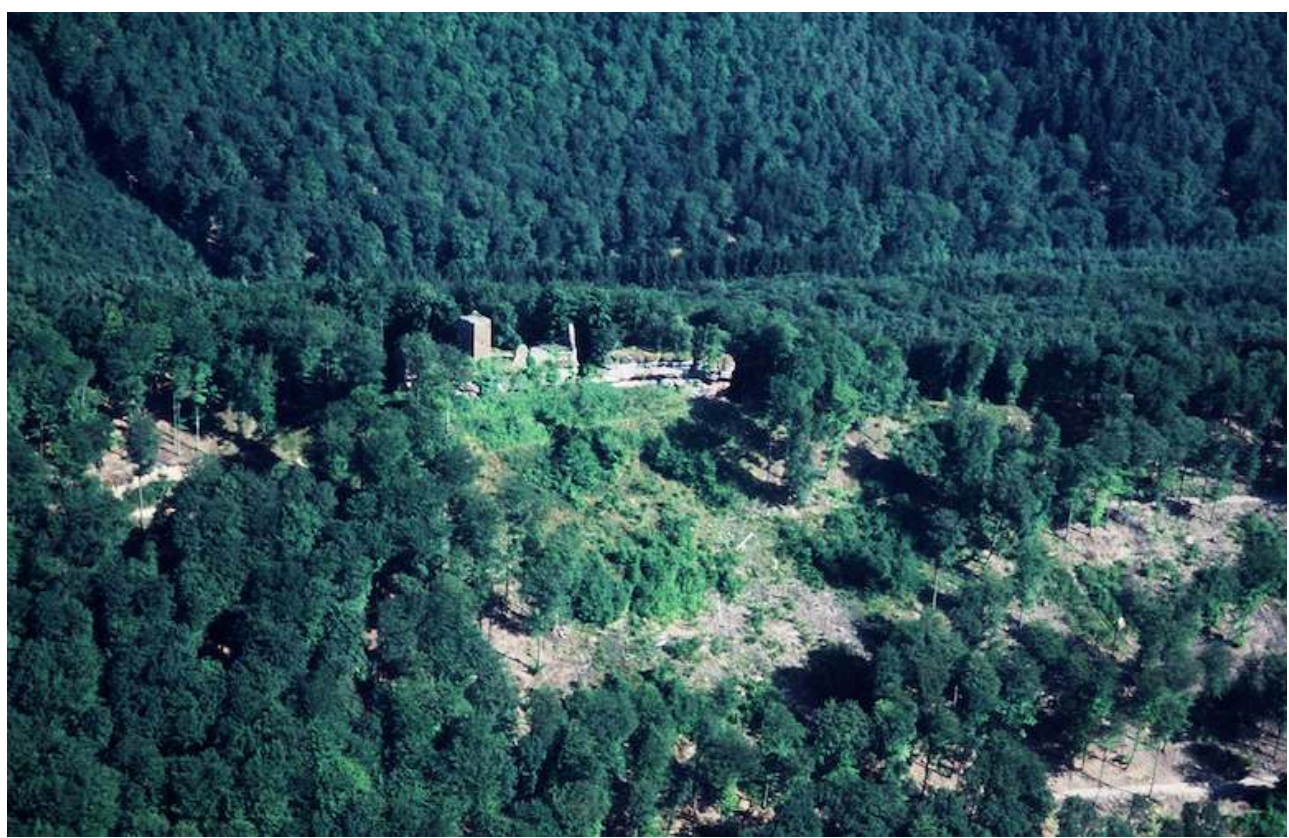

Ruines du château du Falkenstein (Philippsbourg, Moselle) perché sur son éperon gréseux dominant le massif forestier. Les vestiges du château sont totalement démantelés par les troupes françaises de Montclar (1676-1677). La ruine a été remise en scène et valorisée dans la seconde moitié du xix ${ }^{\mathrm{e}}$ siècle.

Source : André Humbert/Cerpa.

\section{Le camp de Bitche}

7 Le siège de Bitche (1870-1871), puis l'annexion de l'Alsace-Moselle suite au traité de Francfort remettent en cause l'organisation du territoire de défense. La ville de Bitche se transforme en ville de garnison avec l'abandon du modèle de la ville-citadelle. Cette conversion s'opère par la construction de grands quartiers militaires et l'arrivée de régiments. Dans un second temps, Bitche accueille un vaste camp de manœuvre à l'est de la ville (figure 3). Ce dernier souligne les dynamiques de la militarisation des territoires par le renforcement des contraintes et des ségrégations entre espaces civil 
et militaire repoussant toujours plus loin ces activités dévoreuses d'espaces (Mathis, 2011).

Figure 3. Une petite ville militarisée aux portes du Pays couvert

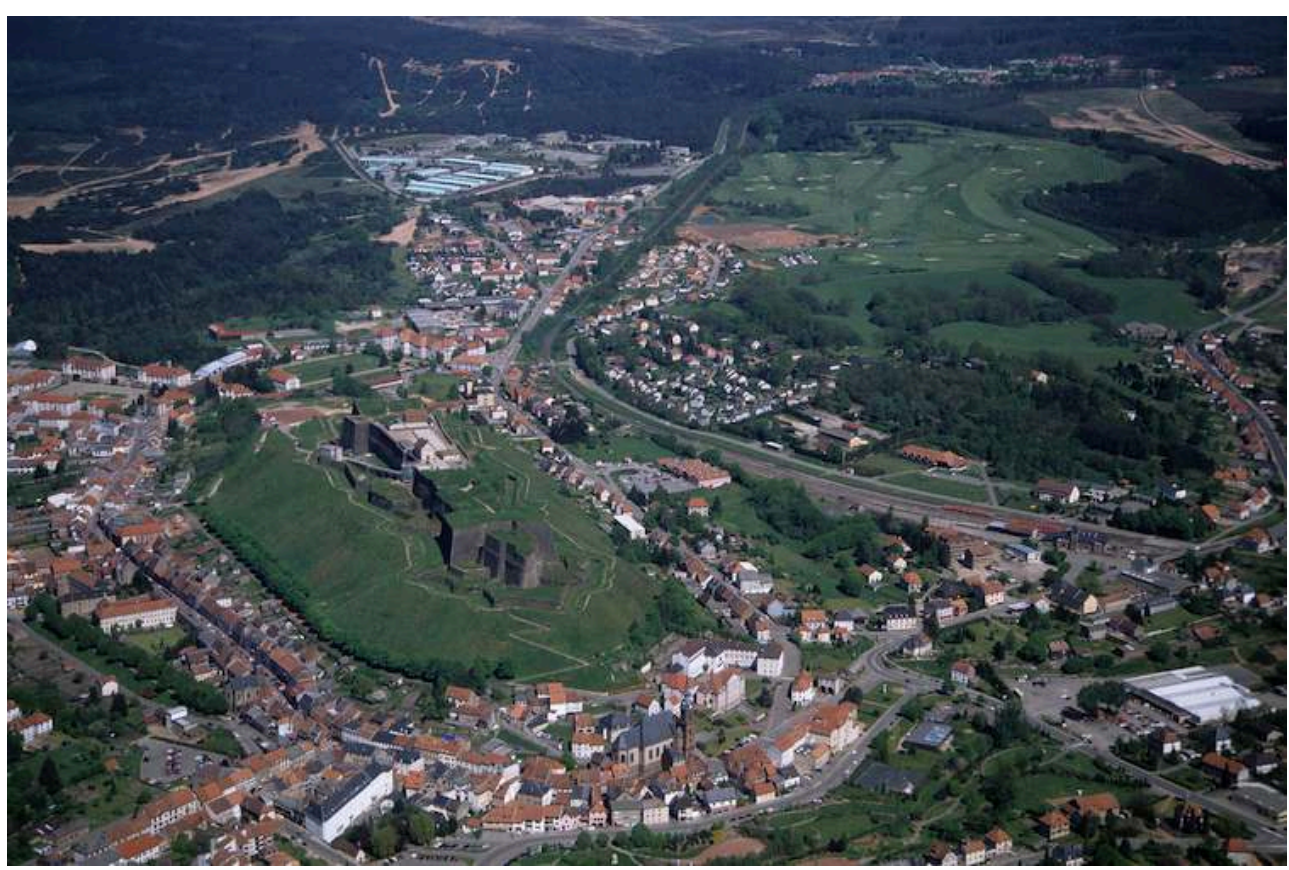

La ville de Bitche s'étend au pied des vestiges de l'ancienne citadelle, ruinée pendant le siège de 1870-1871. À l'arrière-plan, casernes et camp militaire sont adossés à une partie du massif forestier. Source : André Humbert/Cerpa.

8 La construction de camp d'entraînement des troupes est un modèle d'infrastructure qui s'est diffusé au sein de l'Empire allemand (Oberhoffen-sur-Moder en France, Elsenborn en Belgique, Stetten am kalten Markt en Allemagne...). En 1900, à Bitche, l'armée rachète à des particuliers et à la forêt domaniale 3285 ha afin d'établir les infrastructures d'un camp doté d'un terrain de manœuvre. Une voie ferrée forestière d'une longueur de $16 \mathrm{~km}$ relie la gare de Bitche au futur camp afin d'entreprendre les travaux de construction et d'assurer dans un premier temps la vidange des bois. Dès 1901, le service du camp débute. En 1918, l'armée française étend le camp à Ludwigswinkel (Allemagne) pour une capacité d'accueil de 10000 hommes. Cette extension conforte les prétentions territoriales en Sarre et au Bas-Palatinat (Landau). Par la suite, le repli militaire français au-delà de la frontière et la mise en place de la ligne Maginot modifient les fonctions du camp qui devient un casernement de sûreté de la ligne Maginot.

\section{Le déploiement de la ligne Maginot}

Dans le secteur fortifié des Vosges, la militarisation s'est organisée de manière linéaire par des aménagements défensifs le long des cours du Zinselbach, du Rothenbach et du Schwarzbach (Mathis et al, 2017). Ce linéaire défensif de $16 \mathrm{~km}$ de long s'appuyait sur la ligne d'eau, aménagé par d'importants travaux destinés à créer des zones d'inondation, dont la crue était organisée à partir des vidanges des étangs (figure 4). Par ailleurs, le génie a aménagé le cours en un fossé antichar (figure 5). Ce dispositif défensif est protégé par des casemates CORF (Commission d'organisation des régions fortifiées) et 
par des dispositifs de mines (figure 6), enfin les casemates d'artillerie du Windstein et du Grand-Hohékirkel couvrent la vallée.

Figure 4. L'hydrosystème défensif du Rothenbach-Schwarzbach

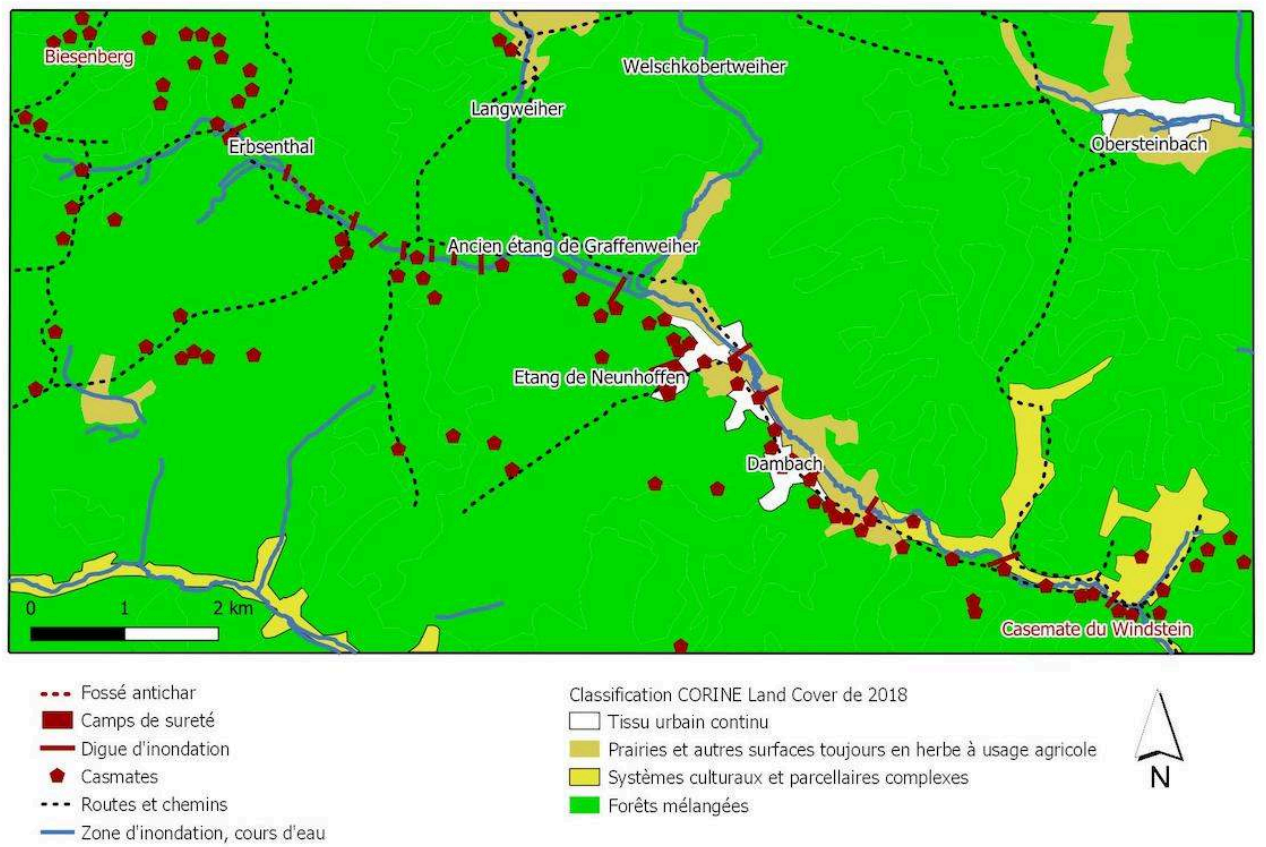

Source : IGN, Remonter le temps. Réalisation cartographique : Tanguy Niederlander.

Enfin, des ouvrages MOM (Main-d'œuuvre militaire), parfois établis en profondeur, couvraient les carrefours des routes forestières aménagés en point d'appui d'ouvrages secondaires. Pour autant, le secteur des Vosges reste le parent pauvre de la ligne Maginot. Abandonné par l'armée dans les années 1960, ce vaste dispositif est resté en partie en l'état. C'est un paysage militaire entré en « congélation historique ». 
Figure 5. Le Rothenbach : un cours militarisé

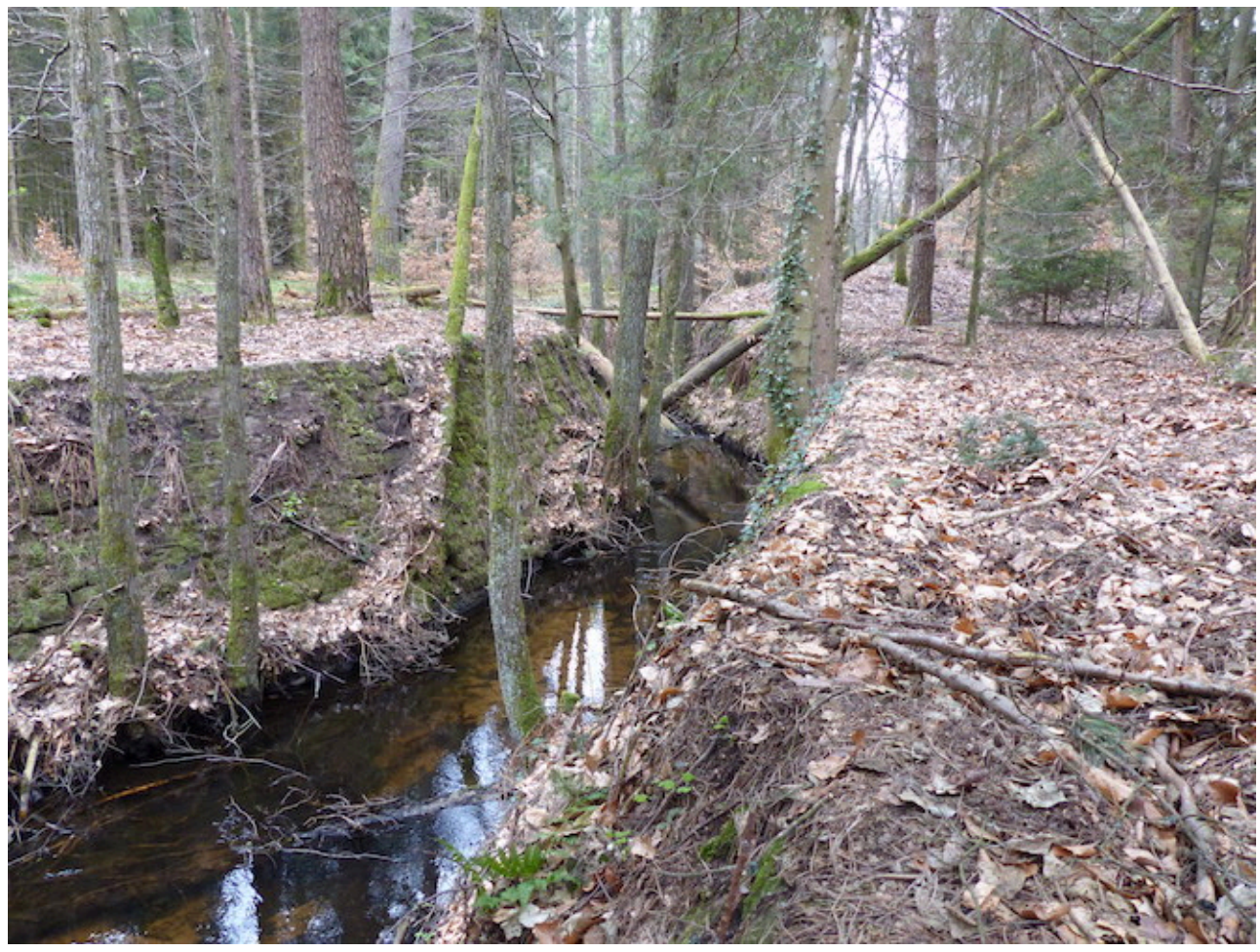

D'une profondeur d'au moins $1,80 \mathrm{~m}$, le cours militarisé du Rothenbach a des berges bétonnées et empierrées. Il était inondé et maintenu en eau par des seuils bétonnés.

Source : Anne Mathis, 2019. 
Figure 6. Dispositif permanent de mines dans la vallée du Rothenbach

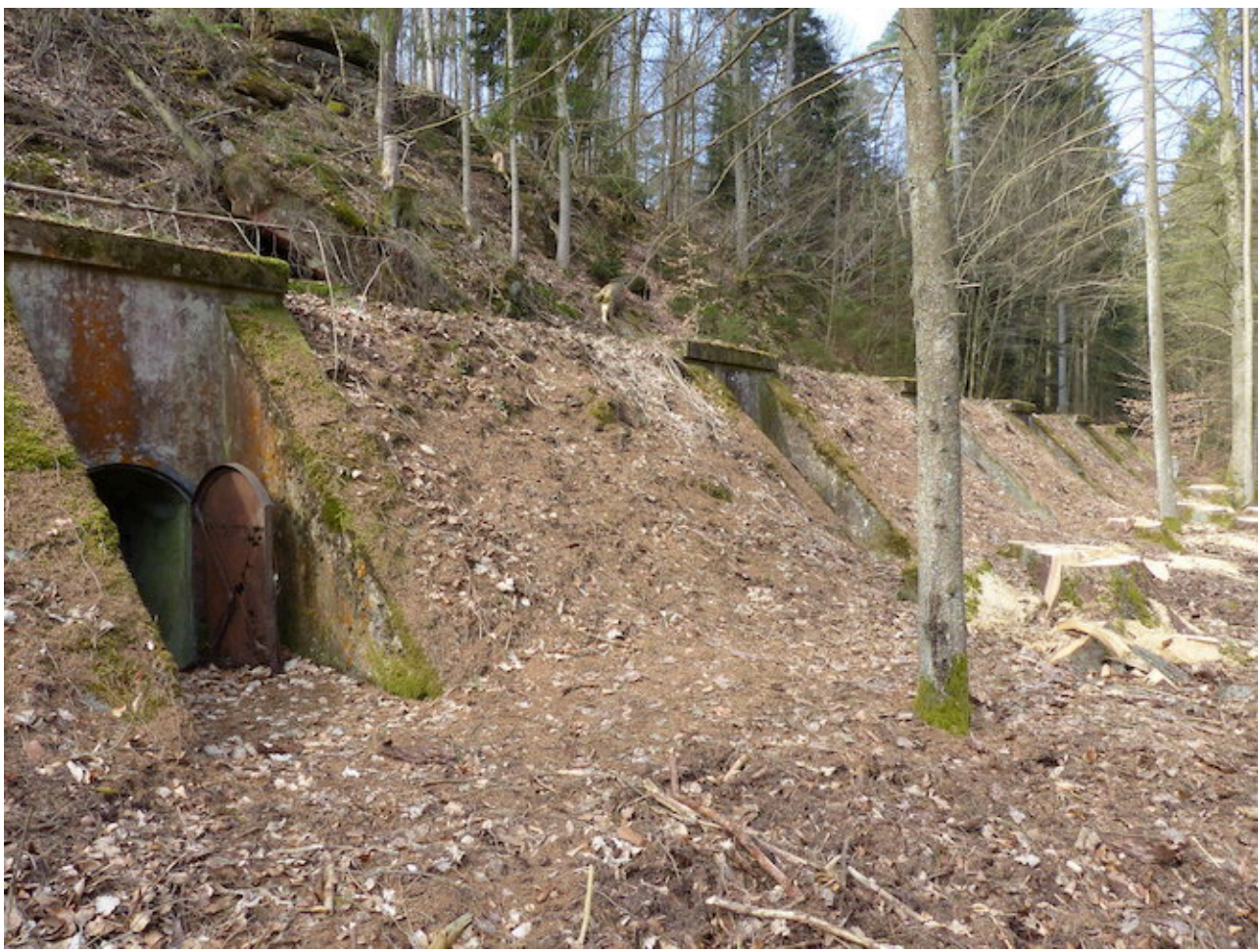

Dispositif de mines permanent, destiné à détruire la route. Les fourneaux mines sont encore en bon état de conservation.

Source : Anne Mathis, 2019.

\section{La forêt de Haguenau}

11 D'une largeur de $30 \mathrm{~km}$, ce massif forestier couvre 21000 ha, avec au cœur la forêt indivise de Haguenau (13 $742 \mathrm{ha})$, à moitié domaniale et à moitié communale. Le massif forestier est au cœur d'un saillant défensif de la ligne Maginot matérialisé au nord du massif par la présence des grands ouvrages CORF (fort de Schœnenbourg). Le nord du massif forestier constitue le dispositif arrière du territoire défensif. En revanche, la ligne principale de défense s'organise dans la partie est du massif sur les terrasses du Rhin. Elle s'appuie sur une zone d'inondation à la confluence de la Sauer, de l'Eberbach et du Klostergraben. L'inventaire mené sur le secteur de la forêt indivise de Haguenau, en vue de son classement en Forêt d'Exception, a permis de recenser plus d'une centaine de traces paysagères du dispositif défensif.

Dans cette portion du massif forestier, un transect paysager des vestiges de la ligne peut être observé :

- dans la partie est, on recense les grands abris CORF (Heidenbuckel, Donau, Soufflenheim) (figure 7) ;

- dans la partie centrale et au nord de la forêt, l'arrière de la ligne principale de défense peut être observé. L'inventaire réalisé a permis d'exhumer les éléments secondaires du dispositif défensif de la ligne Maginot. Organisés en profondeur, ils ont été mis en chantier par la MOM entre 1935 et 1940 et sont organisés pour la plupart en points d'appui afin de contrôler les itinéraires traversant le massif et les points de passage du réseau hydrographique. Ainsi, les points d'appui couvraient toutes les directions. La forêt abritait un quadrillage défensif 
sur 10 à $15 \mathrm{~km}$ de profondeur. Cette structuration des points d'appui montre une organisation caractéristique du champ de bataille fortifié (figure 8).

Figure 7. De grands ouvrages de béton camouflés sous la forêt

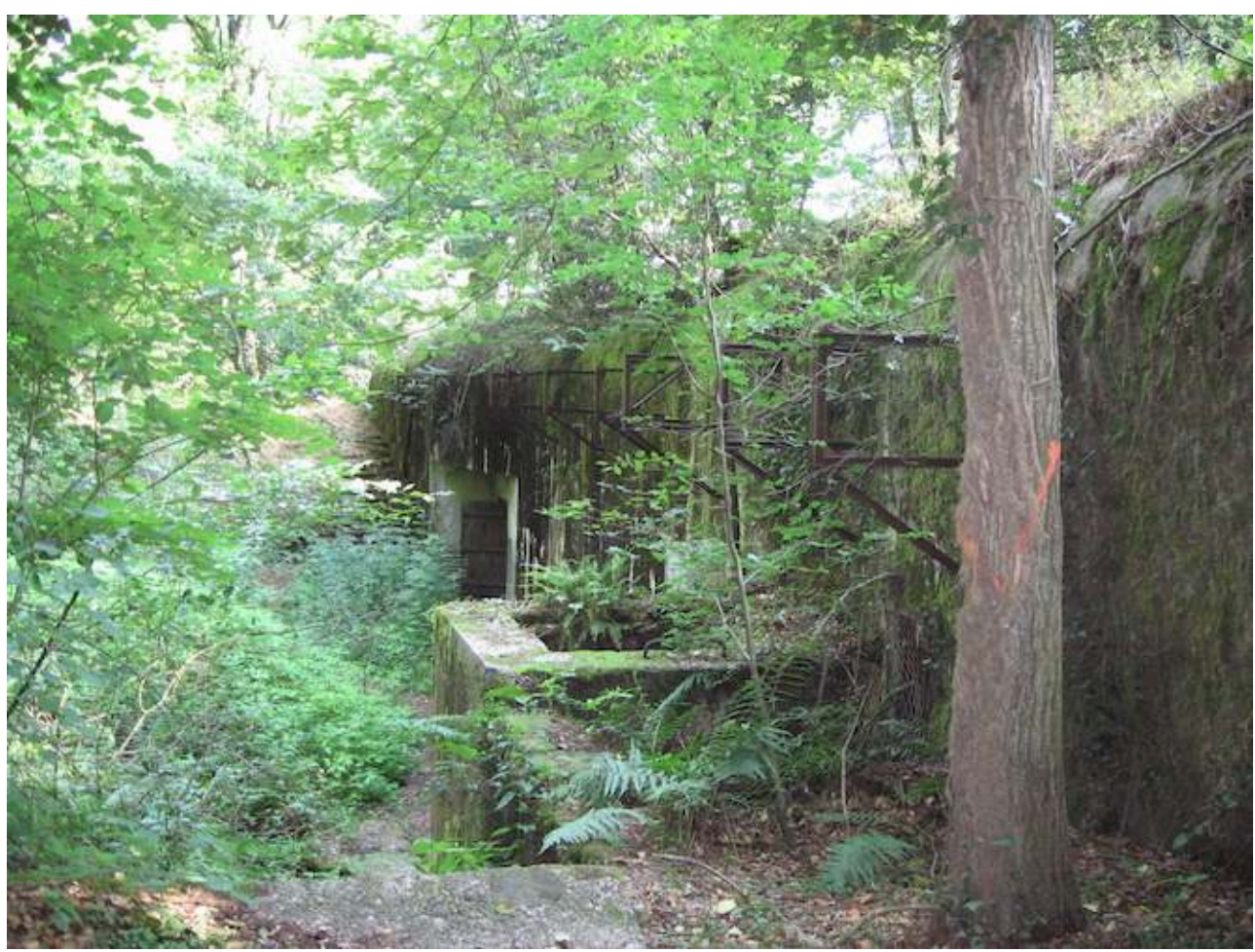

Forêt de Haguenau. Ouvrage CORF de Soufflenheim aujourd'hui camouflé, préservé et fossilisé sous le couvert forestier.

Source : Emmanuel Chiffre, 2019 
Figure 8. Des traces plus modestes de la militarisation

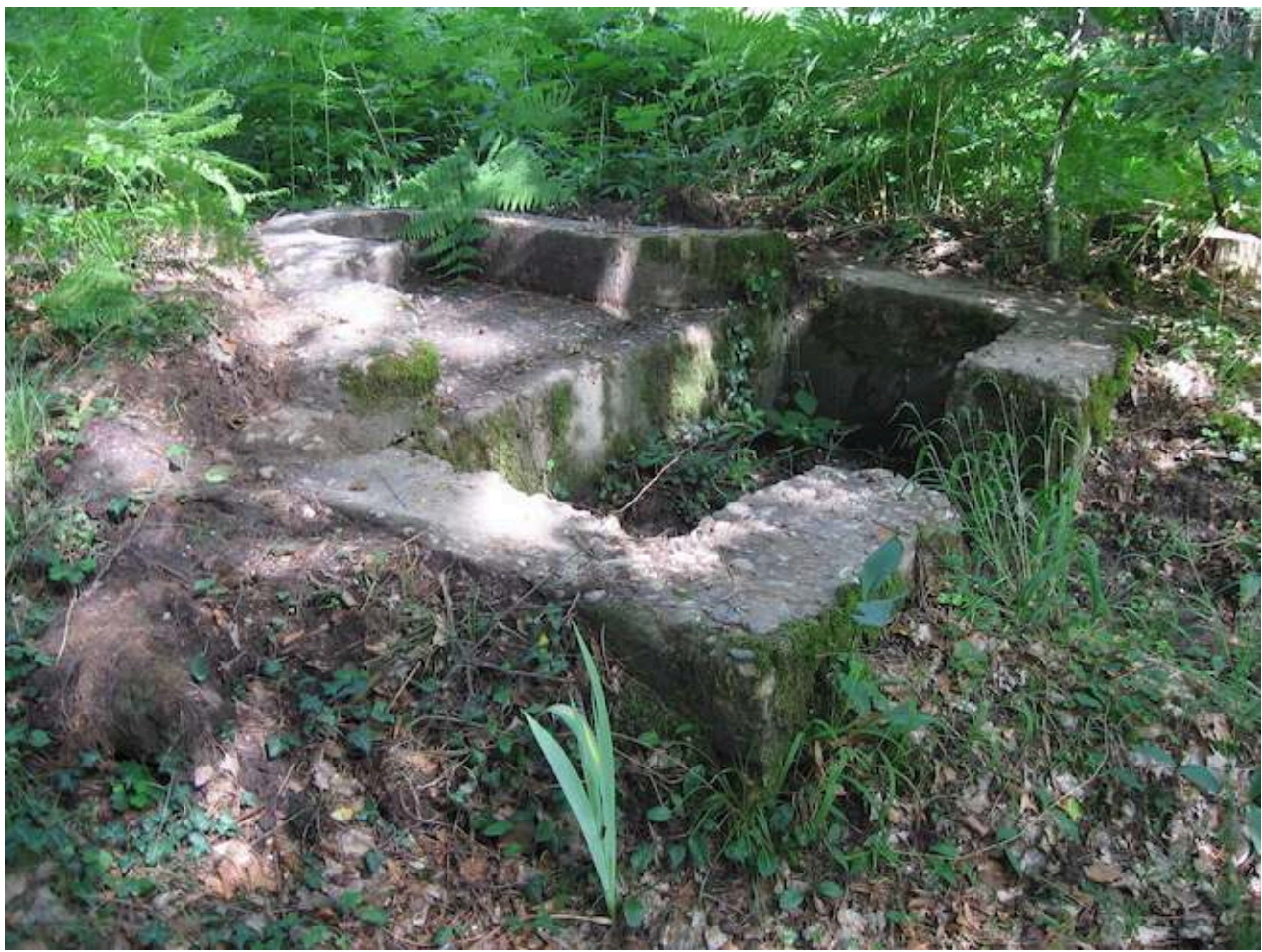

Forêt de Haguenau. Poste de tir aménagé et destiné à protéger les carrefours des allées forestières organisés en points d'appui. Il a été dégagé lors des travaux de prospection et d'inventaire.

Source : Emmanuel Chiffre, 2019.

13 Au sud du massif forestier, l'armée occupe encore le camp d'Oberhoffen-sur-Moder. À partir des années 1887, l'administration allemande agrandit le camp de tir et de manœuvre de Haguenau (1873) par une politique d'acquisitions et de constructions pour établir le camp d'Oberhoffen (environ 1000 ha) sur le modèle des grands camps d'entraînement. Détruit lors de la Seconde Guerre mondiale, il est reconstruit en 1976 et devient le casernement du quartier Estienne. Le territoire du camp reste un espace collectif d'instruction et impose sa réglementation à la gestion de ce secteur forestier.

\section{Le paysage militaire du massif forestier du Bienwald-Mundat}

Le périmètre du massif forestier du Bienwald-Mundat se localise à l'est de Wissembourg dans la vallée du Rhin partagée à cet endroit entre le Palatinat et l'Alsace. La forêt du Mundat désigne en réalité deux régions forestières dont l'histoire est liée à l'abbaye et à la ville de Wissembourg. Nous exclurons de cette étude l'obermundat ${ }^{5}$, à l'ouest de Wissembourg, et retiendrons l'Untermudat ${ }^{6}$ prolongé par le Bienwald.

\section{Les vestiges de la ligne de la Lauter ou de Wissembourg}

C'est dans le massif forestier du Bienwald-Mundat que l'on retrouve le paysage militaire linéaire le plus ancien. Il s'agit de la ligne fortifiée de la Lauter ou de Wissembourg. Construite en 1706, pendant la guerre de Succession d'Espagne (1701-1713), c'est la troisième ligne défensive réalisée par les troupes de Louis XIV, après la ligne de la Queich au nord (1702) et la ligne de la Moder au sud (1703). 
Spatialement la ligne de la Lauter $(36 \mathrm{~km})$ prend appui sur les deux villes fortifiées de Wissembourg et de Lauterbourg. Sur sa rive droite, la Lauter a été bordée d'un important remblai de terre jadis surmonté d'un parapet de tir et de plateformes pour l'artillerie. Le long du cours sur les deux rives, une cinquantaine d'ouvrages (forts, redoutes et demi-lunes) renforcent les fortifications de campagne. La vallée et les abords de la ligne ont été déboisés, la rivière a été aménagée par 28 digues-seuils et 31 écluses en bois. La zone pouvait être inondée artificiellement, sur 300 mètres de large et un mètre de hauteur. C'est donc un hydrosystème défensif (Mathis, 2016) qui a ainsi été construit (figure 9).

Par ailleurs, et malgré la militarisation de la Lauter, le flottage à bûches perdues pouvait se pratiquer jusqu'à Lauterbourg, aussi tous les moulins sur le tronçon Wissembourg-Lauterbourg étaient dotés de pertuis de flottage. Ils étaient également intégrés au sein du schéma de défense. Enfin, la ligne devait être complétée par une place forte moderne bastionnée qui n'a jamais été réalisée : Louisville. La ligne n'a plus été remise en état après les guerres de la Révolution.

Figure 9. La ligne de la Lauter ou ligne de Wissembourg

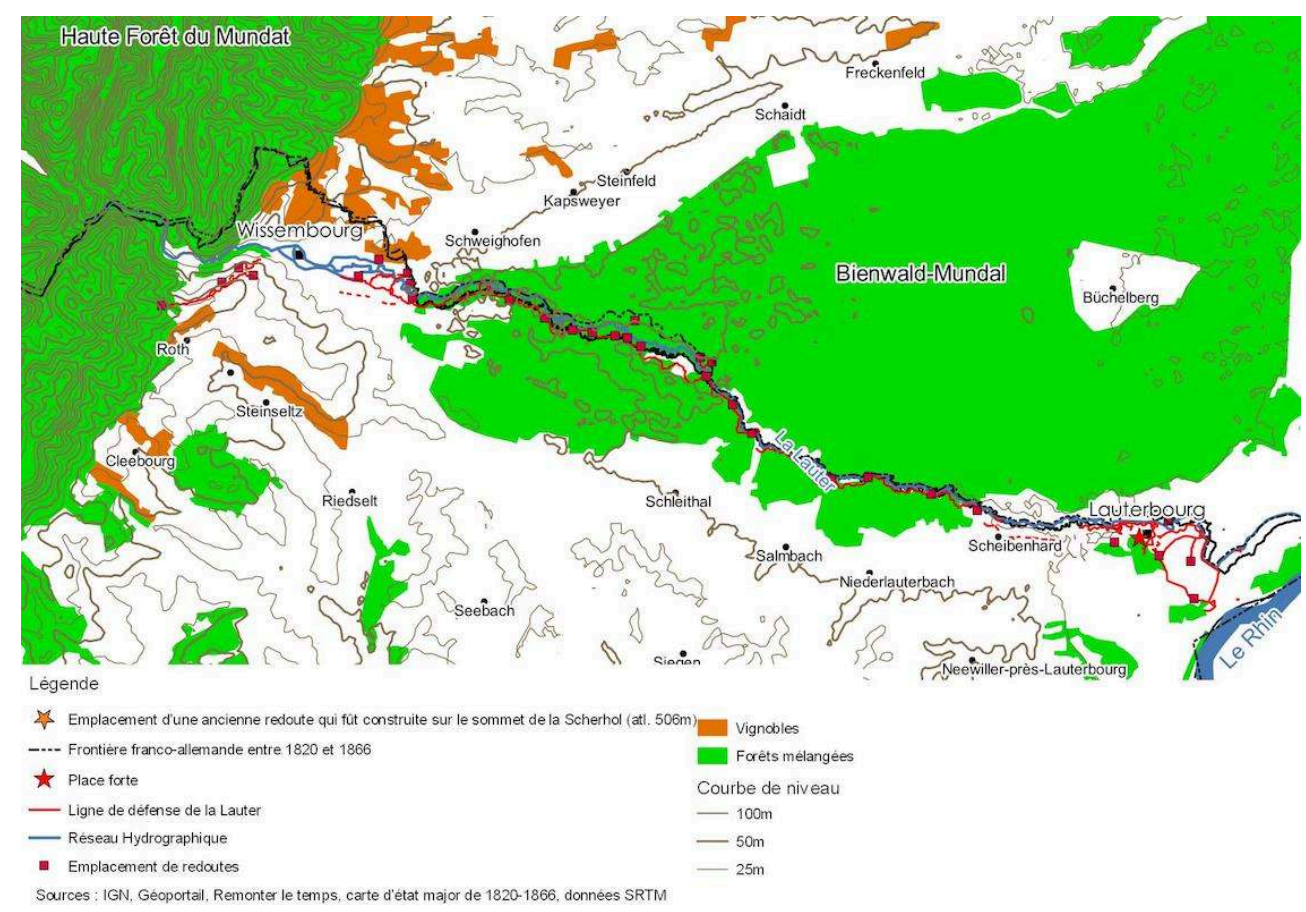

Sources : IGN, géoportail, Remonter le temps, carte d'état-majoir de 1820-1866, données SRTM. Réalisation : Tanguy Niederlander.

\section{Les éléments du Westwall}

Une seconde strate militaire a été déployée au nord de la Lauter durant la période de l'entre-deux-guerres. Il s'agit des éléments défensifs de la ligne principale du Westwall qui s'organisait depuis le Rhin à l'est en direction de Bad Bergzabern et Budenthal dans le massif montagneux du Pfälzerwald. La ligne défensive traversait alors le massif forestier du Bienwald. L'importance de la militarisation peut se quantifier à la densité d'ouvrages construits (172 ouvrages défensifs sur le territoire d'Oberotterbach et 150 ouvrages à Bad Bergzabern). La ligne de défense principale était devancée par des 
réseaux de tranchées et de fils barbelés, d'abattis, de postes d'observation, d'abris de campagne, mais aussi de fossés antichars, de chevaux de frise. La militarisation du territoire frontalier a été particulièrement importante. Cependant, au sein des espaces agricoles ouverts, les bunkers furent détruits et seuls subsistent les éléments de défense linéaire, à savoir les fossés antichars aménagés sur les lignes d'eau (figure 10). La forêt en revanche a conservé les traces ponctuelles de cette ligne défensive, notamment les ouvrages bétonnés qui ont été souvent éventrés lors des combats de 1944-1945 ou lors de sa démilitarisation.

Figure 10. Les vestiges linéaires du Westwall

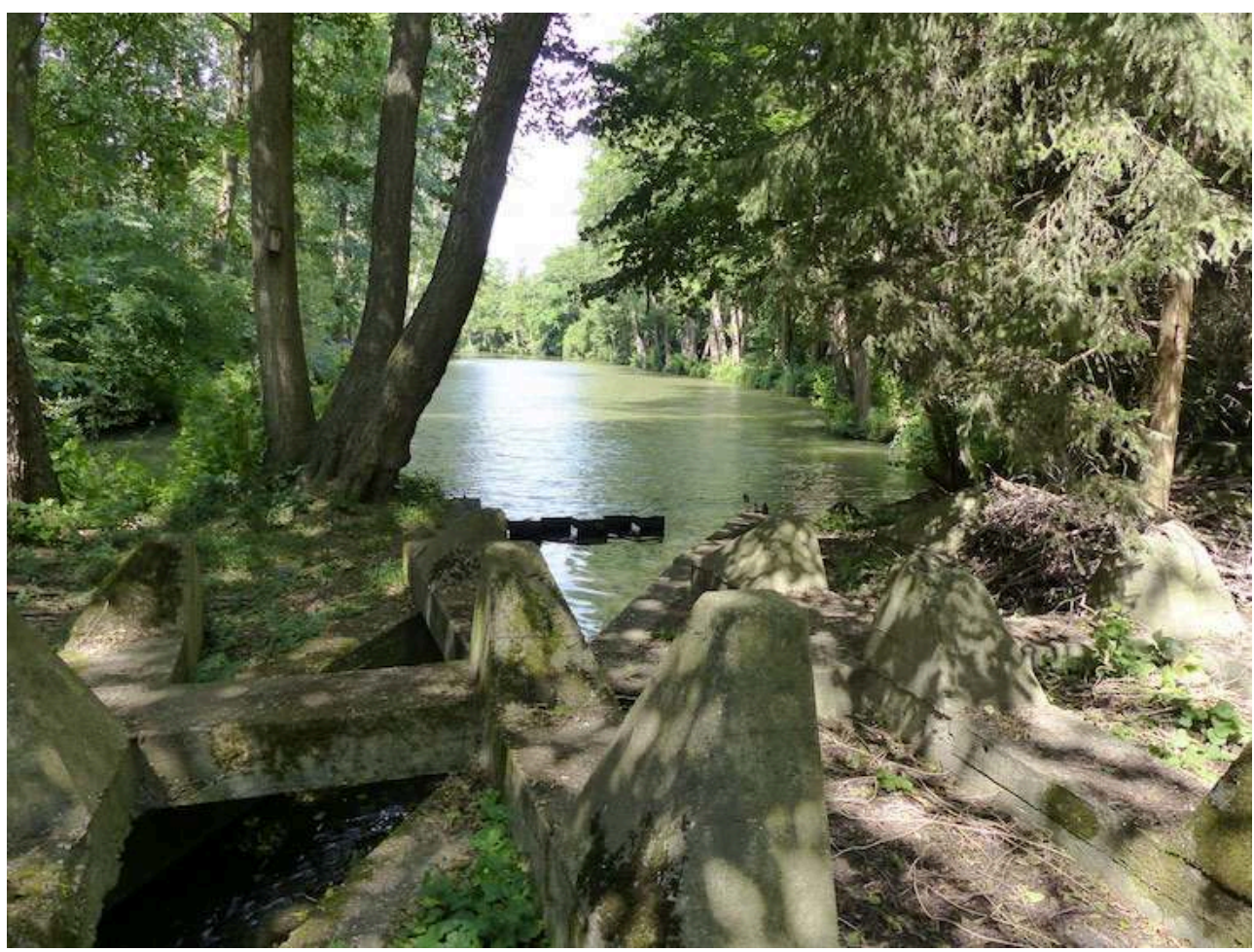

Fossé antichar et dents de dragon sous couvert forestier, des vestiges résilients du paysage militarisé du front défensif.

Source : Anne Mathis, 2016.

Nous venons de présenter la richesse et la diversité des formes de la militarisation. Cependant, il ne s'agit plus que de traces et de fragments de l'ordre du micropaysage. Les lignes défensives avec les ouvrages constituaient un paysage dans une dimension opérationnelle et contraignante, c'est pourquoi il faut considérer que le militarosystème s'est imposé au territoire.

\section{Le paysage militaire sous forêt}

Depuis les travaux de Jean-Pierre Husson (1994), de Jean-Paul Amat (1999), de Jérôme Buridant (2003) ou de Jean-Yves Puyot (2004), les polémopaysages sous forêts ont fait l'objet d'études nombreuses, notamment concernant l'empreinte militaire de la Grande Guerre. Aujourd'hui, la recherche des palimpsestes des polémopaysages détaillés par Frédéric Steinbach et Jean-Pierre Husson (2007) peut être largement étendue grâce à l'outil Lidar. Ce dernier facilite la connaissance de l'empreinte des combats en milieu 
forestier comme le soulignent les travaux de Rémi de Matos Machado et al. (2016) ou de Pierre Taborelli et al. (2017). La question des vestiges militaires en milieu forestier, notamment en l'absence de traces importantes de combats, est restée bien plus confidentielle et limitée (Durup de Baleine, 1998). Si les forêts périurbaines déployées sur l'emprise foncière des grandes ceintures fortifiées commencent à être étudiées, notamment dans le cadre d'une réflexion sur la mise en scène des paysages postmilitaires (Mathis D. et A., 2015 ; Chiffre et Mathis, 2017 ou Pardella et al., 2018), les travaux concernant les forêts militarisées font seulement l'objet d'inventaires et de réflexions (Niederlander, 2019) ${ }^{7}$. On peut supposer que la démilitarisation récente explique ce décalage de temporalité des études entre les forêts marquées par la guerre et les forêts démilitarisées.

\section{La démilitarisation, facteur de " désordre » au sein des paysages forestiers}

indépendamment des massifs forestiers. Dans le processus de militarisation, la forêt n'a pas fait l'objet d'un aménagement et d'une gestion spécifiques, même si en Allemagne, lors de la construction des lignes défensives et des grands camps retranchés (festen), il y a toujours eu une volonté d'intégrer et de conserver une place à la nature dans la mise en œuvre des ouvrages. Cette place était considérée comme le reflet du paysage militaire de l'Empire allemand. Elle était importante dans les représentations du fait militaire. Elle se retrouve dans la mise en scène des ruines des châteaux forts, notamment au sein de l'espace rhénan.

Concernant les territoires fortifiés, le processus de démilitarisation s'est effectué progressivement de 1940 aux années 1970. L'armée a progressivement neutralisé les ouvrages, c'est-à-dire que les armements ont été retirés des postes de combat. Le mobilier et le matériel divers ont été abandonnés à l'intérieur des ouvrages.

Il n'y a eu aucune volonté de la part de l'armée d'un traitement patrimonial à l'exception de l'ouvrage du Simserhof. C'est la raison pour laquelle on peut affirmer que la démilitarisation a été apatrimoniale. Les domaines ont pris en charge la vente des casemates et des forts lorsque ces derniers trouvaient preneurs. Les ouvrages invendus entrent dans un processus dit de «congélation historique ». Les entrées des casemates ont été obstruées mais, la plupart du temps, seules les portes ont été verrouillées. Ce procédé de congélation est typique du démantèlement des lignes fortifiées en Europe (mur alpin en Italie, ligne de la Scanie en Suède...). À l'échelle de la ligne, le dispositif défensif a perdu toute sa fonctionnalité, a été démantelé, laissé à la nature, aux soins des acheteurs ou à la récupération/recyclage sauvage et informel qui « cannibalise » le matériel laissé dans les ouvrages.

Ces traces peuvent se révéler dangereuses par la persistance de champs de barbelés ou d'aiguillons plantés dans le sol (figure 11). Ces lieux insolites et isolés sont devenus des lieux marginaux, qui recueillent graffitis, tags, déchets. Ils sont également des terrains de jeux et de visites, soit de la part de passionnés soit en lien avec des nouvelles pratiques habitantes proches de l'urbex (exploration urbaine), mais aussi du géocaching.

24 Le retour au civil a également pour conséquence l'effacement partiel ou total des vestiges les moins indurés (tranchées, lignes de barbelés, trous individuels). C'est dans 
les espaces forestiers que cet effacement a été le moins opérant (figures 11, 12 et 13). Il est d'ailleurs sensiblement différent d'un massif à l'autre en raison des phases de démilitarisation. Cependant la neutralisation, même partielle, rompt la cohérence du dispositif défensif qu'il devient difficile d'appréhender. Il faut donc considérer que le territoire de défense est devenu un territoire fantôme dont l'empreinte est résiduelle, délaissée et fossile.

Figure 11. Les traces dangereuses au sein des massifs

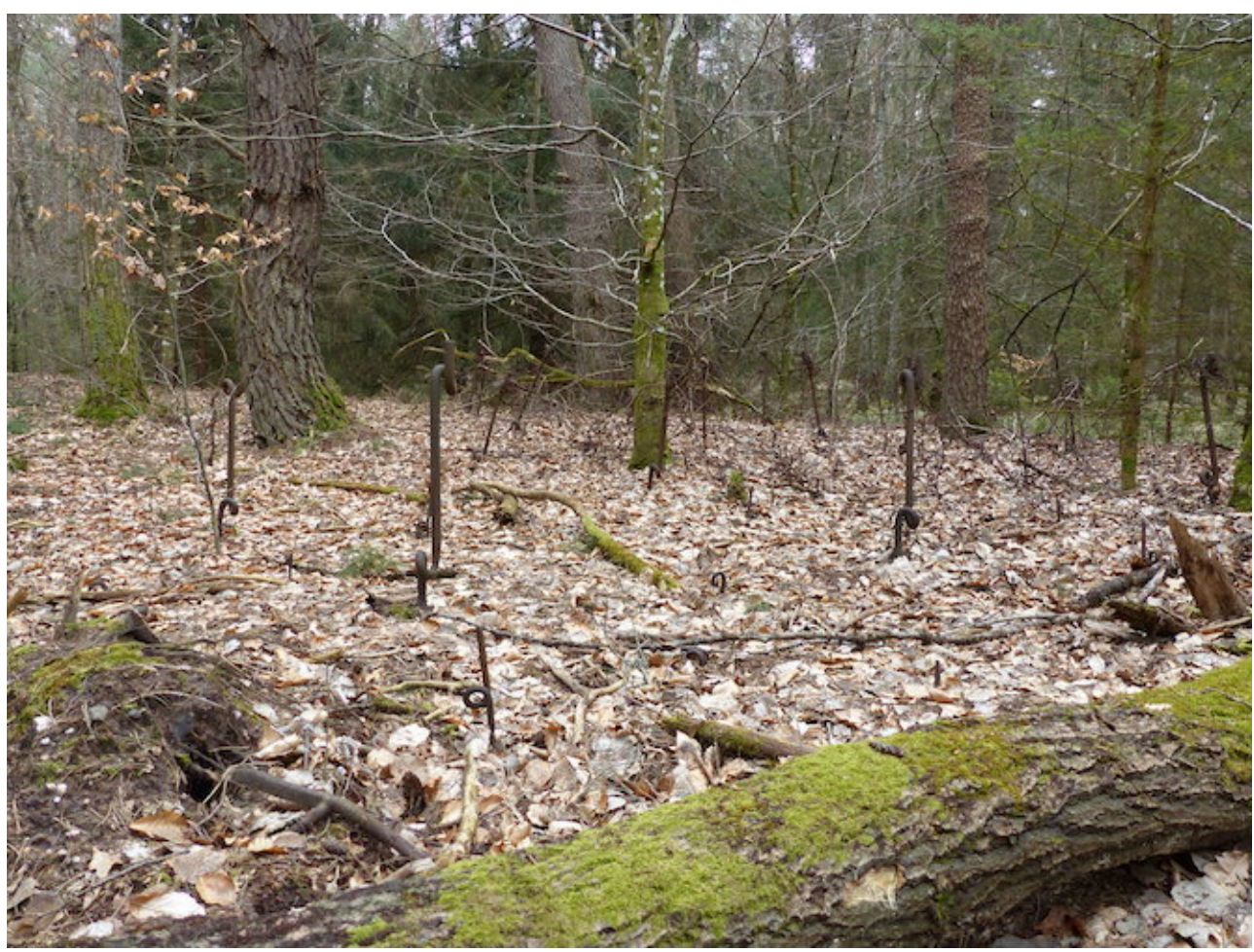

Vosges du Nord. Le vallon du Rothenbach présente encore d'importants éléments liés à la militarisation. Aiguillons, queues-de-cochon et barbelés couvrent encore quelques sections de la rive droite du cours d'eau.

Source : Anne Mathis, 2019. 
Figure 12. Les ruines des casemates neutralisées

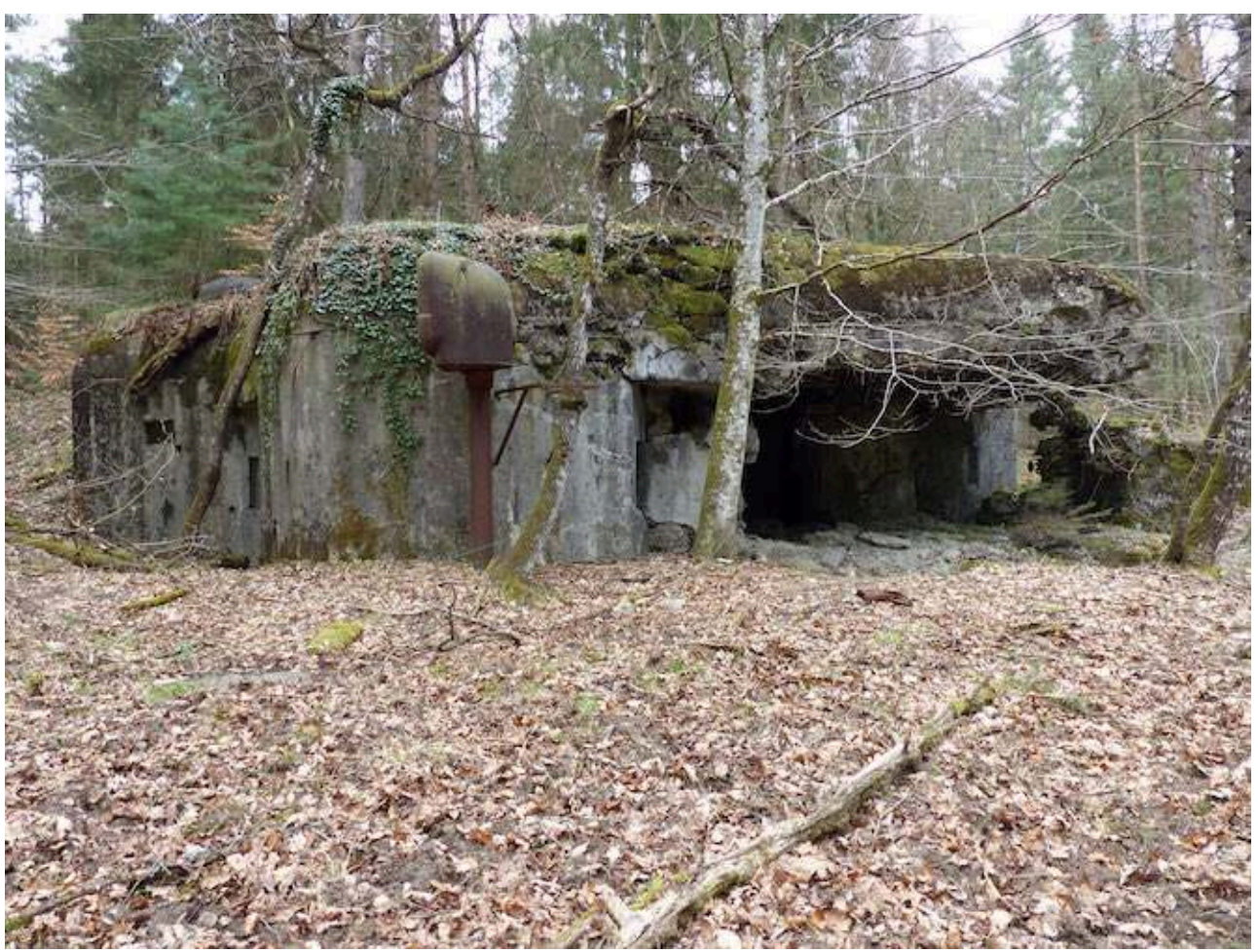

Vosges du Nord. Casemates CORF éventrées et à l'état de ruines.

Source : Anne Mathis, 2019.

Figure 13. Les vestiges de la militarisation des cours d'eau

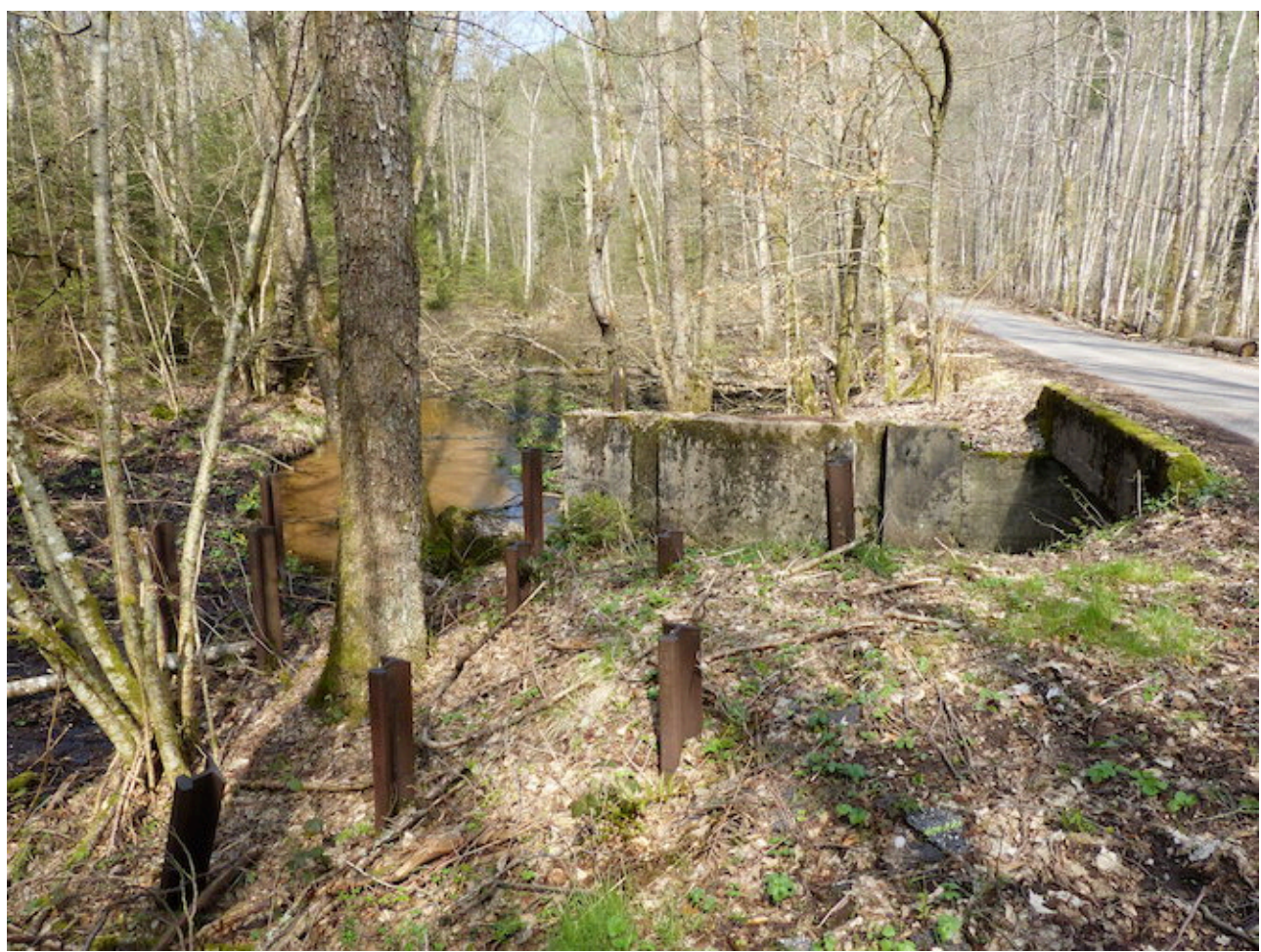

Vosges du Nord. Rails et bief d'inondation soulignent les traces de l'ancien hydrosystème défensif. Source : Anne Mathis, 2019. 
Le départ de l'armée et le processus de démilitarisation permettent de soutenir les démarches de renaturation par l'effacement physique ou simplement informatif des éléments militaires. La vallée de la Lauter de Wissembourg à Scheibenhard est présentée « comme l'une des rares rivières de plaine à avoir conservé des eaux propres et un lit nature ${ }^{8} "$, alors que nous avons vu que le cours avait été aménagé pour asseoir la défense de la ligne de la Lauter mais aussi pour assurer le flottage du bois. Même si la militarisation est connue, s'appuyant sur le classement au titre des monuments historiques, notamment le fort Saint-Rémy à l'est de Wissembourg, ce classement n'instaure pas de protection.

Projets de paysage, 22 | 2020 
29 Dans le secteur de Bitche, la renaturation des cours d'eau mise en œuvre sur le Rothenbach et le Schwarzbach cherche le plus souvent à effacer les vestiges dans une logique de reconstruction d'un paysage dit naturel. Travaux de reméandrage, effacement d'étangs, dégagement du cours des obstacles militaires cherchent à reconstruire un paysage rivulaire naturel dans une logique d'amélioration de la qualité des eaux de la partie aval des bassins-versants. Cette démarche soutenue et encadrée par le Parc naturel régional des Vosges du Nord et des associations de bénévoles cherche à effacer l'impact environnemental de la militarisation des cours d'eau. La renaturation environnementale du cours d'eau et la volonté d'effacer ou d'atténuer l'empreinte militaire expriment une approche apatrimoniale de l'héritage militaire.

Les vestiges militaires, bien qu'imbriqués à l'espace forestier, sont considérés comme un élément qu'il convient d'atténuer sinon d'effacer. L'absence d'inventaire complet et la non-sécurisation des lieux témoignent de la volonté de ne pas prendre en compte cet espace au sein du sylvosystème. Il s'agit bien d'une démarche qui vise à exclure l'empreinte militaire d'un paysage rivulaire et forestier considéré comme naturel (Znieff et Natura, 2000) ${ }^{9}$. L'approche est comparable à la gestion des massifs forestiers marqués par la guerre, le retour au civil est ainsi une opportunité pour restaurer la « nature ». Dans ce cas il s'agit d'une « nature reconstruite » et remise en scène. En fait, l'armée met en avant sa gestion environnementale des tourbières, des landes et des forêts du camp de Bitche situé dans la partie lorraine des Vosges du Nord classée réserve mondiale de biosphère par l'Unesco. L'armée s'inscrit dans une politique qui correspond à la gouvernance environnementale des Vosges du Nord. L'empreinte militaire ancienne, parce qu'elle a transformé le milieu, est jugée perturbatrice.

\section{Les massifs forestiers militarisés : un paysage culturel}

31 Toutefois, qu'il s'agisse aujourd'hui des massifs de Bitche dans le cadre de l'OHM Pays de Bitche, de la forêt du Mundat, ou de la forêt indivise de Haguenau, la question du paysage forestier militarisé commence à s'inscrire dans une approche très similaire à celle des "paysages culturels» définis outre-Rhin comme concept directeur de l'aménagement des espaces en Allemagne (Schenk, 2013).

32 Cette notion de paysage culturel est informative et permet une prise de conscience collective d'un héritage qui caractérise les massifs forestiers dans leur organisation et leur gestion. Elle peut développer, de manière cumulative, une logique de conservation mais aussi de planification dans une démarche à la fois rétrospective, liée à la géohistoire, mais aussi prospective, visant à développer des outils de gestion et d'aménagement global. Aussi, elle facilite la définition même, à partir d'un paysage plus ou moins fossilisé, d'éléments ressources au sein d'un processus de conversion. C'est cette approche que sous-tendent les démarches actuelles qui deviennent tout autant support à la production identitaire, construisant un bien collectif avec une exigence de qualité paysagère.

33 La démilitarisation modifie donc la lecture de l'ancien paysage militaire. Cette transformation conduit à distinguer plusieurs approches qui conditionnent les démarches de réappropriation. Le paysage peut ainsi être considéré comme fossile, informateur et culturel. Il faut noter que pour les trois massifs forestiers il n'y a pas réellement de place pour une dimension commémorative du paysage militaire. 


\section{Une multiplicité des prises en compte de l'héritage militaire sous forêt}

\section{Une strate paysagère qui ne peut plus être ignorée}

Contrairement aux polémopaysages qui ont contraint à une gestion spécifique par les forestiers au lendemain de la Première Guerre mondiale, la strate militarisée des paysages forestiers a longtemps été privée de toute démarche d'appropriation. Aujourd'hui, cette strate militaire fossile ne peut plus être ignorée parce qu'elle fait l'objet de pratiques habitantes renouvelées (abris et points de ralliement de chasseurs, espaces d'expression squattés, terrains de jeux alternatifs pour enfants et adultes...). La multiplicité des trajectoires des massifs forestiers se révèle progressivement dans les formes d'appropriation de l'héritage militaire. Refusés, conservés ou valorisés, voire patrimonialisés, les délaissés de l'armée sont effacés ou entrent en scène dans les formes de politique de gestion de la forêt. La valeur de l'héritage militaire n'est pas liée à la qualité des vestiges mais au contexte de mise en scène et à la manière dont les acteurs (armée, propriétaires privés, Office national des forêts, municipalités...) envisagent la place que ces derniers doivent prendre dans les massifs. Cela relève de la perception paysagère de la friche militaire mais aussi des espaces forestiers.

Comme le rappelle Benoît Boutefeu (2009), «la compréhension du processus de patrimonialisation, qui fait de la forêt un bien commun, constitue une clef d'entrée pour aborder les différentes sensibilités des acteurs forestiers et mener une véritable gestion patrimoniale ». Cependant, cette gestion patrimoniale n'implique pas une trajectoire unique. En fonction des projets de territoire, la valorisation ou non de l'héritage militaire n'est qu'une possibilité. Le paysage militaire n'est qu'une ressource au sein du massif. Elle peut s'inscrire dans des temporalités différentes.

La ressource "fossile » est une jachère qui peut être mobilisée dans le cadre d'une gestion territoriale des massifs. Mais cette mise en scène s'inscrit dans un contexte local, régional ou national particulier. Ainsi les châteaux du Pays de Bitche, et plus globalement du versant alsacien et mosellan des Vosges, ont fait l'objet d'un soin tout particulier du Club vosgien. Il s'agissait alors d'une mise en scène paysagère qui accompagnait la recherche de belvédères offrant des panoramas, ouvrant sur des horizons lointains et des frontières perdues, formes nostalgiques d'une frontière au Rhin ou d'une frontière repoussée. Si aujourd'hui la difficulté de sécuriser ces sites contraint à en limiter l'accès, ces derniers sont une ressource en jachère qui peut être mobilisée. Pour Lucie Dupré (2008): «Le site - dans son extension paysagère, touristique, archéologique, pittoresque qui peut être la ruine médiévale aussi bien que la friche et quel que soit le degré de clarté et d'officialité de son statut - est un espace, un support et un produit de patrimonialisation hybride. Il annonce une présence humaine passée ou à venir... »

Plus globalement, toutes les ruines militaires sont désormais en cours d'inventaire, devenant un enjeu d'aménagement du territoire. Ce travail d'inventaire, en l'absence de Lidar, passe nécessairement par le travail de terrain afin d'assurer géolocalisation, identification et cartographie qui permettront ensuite d'asseoir toutes les démarches ultérieures. L'inventaire permet de respatialiser des vestiges anciennement abandonnés et d'effectuer des choix territoriaux portant sur la sécurisation, la valorisation, la conservation ou la mise en jachère de lieux dans le cadre d'une gestion 
globale du massif forestier. Réalisé sur les éléments du Westwall, ou sur la ligne Maginot en forêt d'Haguenau (dossier de labellisation en Forêt d'Exception), il est très partiel dans le massif de Bitche (OHM Pays de Bitche). Tout reste à entreprendre en forêt du Bienwald-Mundat concernant la ligne de la Lauter.

\section{Quelles prises en compte de l'héritage militaire sous forêt?}

\section{Les démarches de labellisation : la forêt indivise de Haguenau}

Dans le cadre de la gestion plus globale et complexe des massifs forestiers et notamment pour traduire une volonté d'une gestion multifonctionnelle, les démarches de labellisation des forêts domaniales illustrent la volonté d'appréhender les massifs suivant des enjeux durables et comme une ressource globale dans une politique de mise en valeur plus ambitieuse que la seule gestion sylvicole du massif. La labellisation Forêt d'Exception s'inscrit dans une démarche de mise en réseau des forêts domaniales. Elle permet de construire autour d'un projet global la gestion des massifs en fédérant les acteurs mais aussi de conforter l'affirmation d'un «patrimoine naturel» et d'une ressource, en inscrivant la forêt dans une histoire et un récit tels que la militarisation. Depuis 2008, 17 forêts, choisies parmi les forêts domaniales gérées par l'ONF sur l'ensemble du territoire, sont officiellement engagées dans la démarche Forêt d'Exception. Cette démarche de labellisation de la forêt indivise d'Haguenau a été signée le 30 janvier 2020. Elle souligne une trajectoire du massif forestier qui intègre, à sa gestion territoriale, des territorialités héritées dont un des volets est centré sur l'important héritage militaire. Ce dernier a été intégré au projet de développer une offre de loisirs nature de qualité pour améliorer l'attractivité du massif. L'héritage militaire trouve sa place dans la mise en chantier de sentiers de découverte multithématiques. Cette démarche implique de la part des acteurs (ONF, communes) de sensibiliser les visiteurs à la découverte et à la préservation tout en consolidant les liens avec le territoire. Il s'agit de considérer le massif comme un espace partagé dont il faut prendre soin. La démarche se veut pédagogique auprès d'usagers de la forêt et doit permettre de faire prendre conscience de la diversité du sylvosystème, en mettant en avant les éléments "fossiles » de l'ancien militaro-système. Le récit militaire n'est qu'une composante d'un récit global d'une géohistoire du massif forestier qui est le porteur de projet territorial (Sgard, 2008).

\section{Une vision duale du massif frontalier de Mundat-Bienwald}

Comme nous l'avons précisé précédemment, le massif est complexe, intégrant tout à la fois des vestiges du Westwald et des vestiges de la ligne de la Lauter. Cependant on observe deux approches différentes au sein du massif. Stimulé par le Bund für Umwelt und Naturschutz Deutschland ou Bund ${ }^{10}$, le regard sur les espaces militarisés a évolué. Dès les années 1970, le Bund a signalé l'espace naturel et la ceinture verte que représentait le rideau de fer. Il milite également sur la valeur écologique et environnementale de l'ancien Westfall, argumentant sur le fait que les ruines des bunkers se sont transformées en habitats écologiques. La disposition linéaire des ruines constitue un corridor dans un réseau de biotopes. Aujourd'hui, transposées à l'ancien Westwall, ces approches permettent d'envisager l'ancienne ligne fortifiée comme le "mur vert à l'ouest ». Aussi, le Bund a été un acteur influent dans les démarches de réévaluation des délaissés des installations militaires. La démarche proposée cherche 
tout autant la conservation de la nature que la protection des monuments. Elle s'appuie désormais sur la loi sur la conservation du 6 octobre 2015, qui facilite les synergies entre la protection de la nature et celle du patrimoine culturel, relayées par le projet Grüner Wall im Western. Le paysage militarisé trouve sa place au sein des espaces forestiers mais plus globalement à l'échelle d'un vaste corridor frontalier. La démarche paysagère est globale et intègre toutes les composantes. Ainsi, dans le désordre d'un tiers paysage qui constitue la strate inférieure du paysage du massif forestier du Mundat se dessine cette approche des paysages culturels.

Il convient de nuancer le propos car le long de la Lauter, les vestiges de la ligne défensive ne sont pas pour l'instant intégrés à la trajectoire de valorisation. Seuls les moulins et quelques biefs d'inondation font l'objet d'un affichage pédagogique. Les redoutes restent à l'état d'artefact. Sur la rive droite de la Lauter, le fait militaire a été minoré. Il n'y a pas d'affichage pédagogique, pas de signalisation hormis environnementale. Les fortifications ne sont pas repérées et servent de supports aux activités de loisirs sportifs (VTT). La gestion de l'héritage militaire est totalement absente du territoire.

\section{Questionner la place de la militarisation au sein du milieu forestier}

41 En forêt de Bitche, malgré (et peut-être à cause de) la présence de l'armée, la question de l'héritage militaire n'est pas encore totalement entrée dans la valorisation territoriale au sein du massif. Cela peut paraitre paradoxal si on considère que l'empreinte militaire - qu'il s'agisse de l'imposante citadelle et de l'ouvrage du Simserhof - représente une ressource déjà valorisée, ce qui permet de construire un projet de tourisme militaire (Chiffre et al., 2020). En revanche, la militarisation sous couvert forestier est encore peu mise en valeur, reste en jachère et fait l'objet d'un lent processus d'effacement et d'altération. Dans les représentations de certains acteurs, le paysage environnemental des Vosges du Nord doit effacer ou nier la part anthropique, qu'il s'agisse d'ailleurs d'héritages militaires ou stagnustres et tend à rechercher un état d'origine du milieu. Aujourd'hui, cette démarche fait débat au sein du territoire et des habitants. 
Figure 14. Approches rétroprospectives de la ligne Maginot, supports du récit

\begin{tabular}{|c|c|c|c|c|c|c|c|}
\hline & $\begin{array}{l}\text { Structures et territoires de } \\
\text { défense }\end{array}$ & & Représentation & & Paysage et panorama & Faciès forestier & Géosymbole \\
\hline $\begin{array}{l}\text { Avant- } \\
\text { guerre }\end{array}$ & $\begin{array}{l}\text { Ouvrages CORF } \\
\text { Réalisation des obstacles } \\
\text { normalisés } \\
\text { (Fossés antichars, zones } \\
\text { d'inondation, champs de rails...) }\end{array}$ & 2 & $\begin{array}{l}\text { Ligne, symbole de } \\
\text { dissuasion, de sécurité, de } \\
\text { protection } \\
\text { Modernité de l'ingénierie } \\
\text { militaire, géographie } \\
\text { militaire (topographie) } \\
\text { Secret militaire }\end{array}$ & r & $\begin{array}{l}\text { Anticipation du champ de } \\
\text { bataille } \\
\text { Camps de sûreté (village } \\
\text { militaire - couple } \\
\text { village/garnison) } \\
\text { Paysage agro/militaire }\end{array}$ & $\begin{array}{l}\text { Espace stratégique } \\
\text { Obstacle dans la progression, } \\
\text { camouflage } \\
\text { Paysage forestier participant } \\
\text { au mythe d'une ligne } \\
\text { continue de fortifications }\end{array}$ & $\begin{array}{l}\text { Symbole de la puissance } \\
\text { militaire (fort du } \\
\text { Simserhof) } \\
\text {, Frontière inviolable } \\
\text { Drôle de guerre }\end{array}$ \\
\hline Guerre & $\begin{array}{l}\text { Remplissage intercalaire par les } \\
\text { ouvrages MOM } \\
\text { Front continu organisé en } \\
\text { profondeur } \\
\text { Destruction/déconstruction } \\
\text { pendant la période de } \\
\text { l'Occupation (« cannibalisation » } \\
\text { des infrastructures pour réaliser } \\
\text { le mur de l'Atlantique) }\end{array}$ & $>$ & $\begin{array}{l}\text { Evacuation des populations } \\
\text { civiles } \\
\text { Combats secondaires } \\
\text { destinés à percer la Ligne } \\
\text { (Sarre- Rhin - Vosges), } \\
\text { démontrer la supériorité } \\
\text { militaire de l'Allemagne }\end{array}$ & $>$ & $\begin{array}{l}\text { Champ de bataille aménagé } \\
\text { Paysage de l'invisible et } \\
\text { souterrain des grands } \\
\text { ouvrages et des postes de } \\
\text { combat } \\
\text { Destruction des forts et des } \\
\text { villages bordiers }\end{array}$ & $\begin{array}{l}\text { Rôle mineur dans les } \\
\text { opérations militaires }\end{array}$ & $\begin{array}{l}\text { Effondrement du mythe } \\
\text { de la frontière } \\
>\quad \text { Symbole de la défaite de } \\
1940\end{array}$ \\
\hline $\begin{array}{l}\text { Après- } \\
\text { guerre }\end{array}$ & 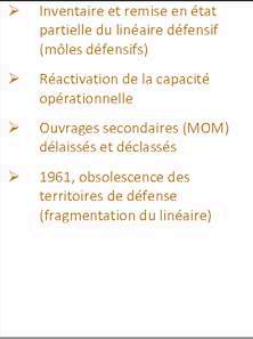 & 7 & 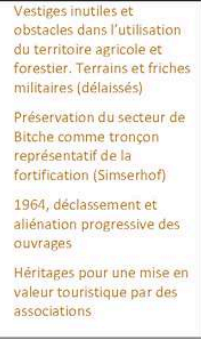 & $>$ & $\begin{array}{l}\text { Paysage de la friche, paysąe } \\
\text { mareinal, paysage effacé } \\
\text { (archéologie militaire) } \\
\text { Paysage recyclé (étangs + } \\
\text { sites de visites + } \\
\text { muséographie..) } \\
\text { 1969, inventaire } \\
\text { photographique de la Ligne } \\
\text { Maginot par I'Etablissement } \\
\text { cinématographique et } \\
\text { photographique des Armées } \\
\text { (ECPA) }\end{array}$ & 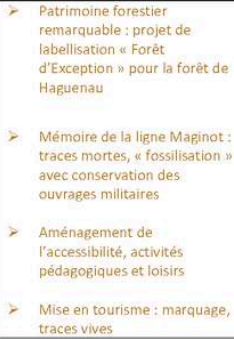 & $\begin{array}{l}\text { Géosymbole d'une } \\
\text { histoire militaire globale } \\
\text { al'échelle européenne } \\
\text { des grandes lignes } \\
\text { défensives de l'Entre- } \\
\text { deux-guerres }\end{array}$ \\
\hline
\end{tabular}

Source : d'après Emmanuel Chiffre et al. 2020.

L'affirmation d'un tourisme militaire qui régénère un territoire en marge doit intégrer les nouvelles pratiques (géocaching, urbex) et assurer la sécurisation dans un massif parcouru par les randonneurs qui ont déjà conquis les ruines des châteaux et, clandestinement, les vestiges de la ligne Maginot. Ces pratiques habitantes soulignent l'intérêt d'assurer la gestion de l'héritage militaire au sein des massifs forestiers.

Tenir compte de la reconstruction du récit de la militarisation passe donc aujourd'hui par un travail d'inventaire de la multitude des ouvrages abandonnés par l'armée. Ce massif forestier, par la variété des formes de la militarisation mais aussi par leur état, permet d'établir un récit rétroprospectif de territoires militaires fantômes (figure 14).

\section{Conclusion}

À travers l'étude de l'empreinte militaire au sein de trois massifs forestiers, de la présentation de l'important patrimoine militaire sous forêts, vestige et témoin de la construction territoriale de la frontière, nous avons montré les bases d'un paysage culturel complexe. La démilitarisation progressive est apatrimoniale, elle a entraîné une "congélation historique ", synonyme de fossilisation du paysage. Ainsi, la forêt passe du statut de "confins militaires" (Boulanger, 2010) à celui de "confins démilitarisés » fossiles. Ce dernier est en réalité en jachère longue susceptible d'être revalorisée.

Aujourd'hui, la prise en compte croissante des patrimoines naturels et culturels dans la gouvernance des massifs forestiers favorise la remise en scène à des degrés divers des éléments de ce paysage militarisé conduisant à des démarches de labellisation. Mais cette prise en compte reste encore partielle et fragmentée. Dans une logique de construction d'un rapport « homme-milieu » renouvelé, les paysages culturels sont des facteurs de production d'identités nouvelles qui permettent d'individualiser des trajectoires de gestion territoriales des massifs forestiers. 


\section{BIBLIOGRAPHIE}

Amat, J.-P., « La forêt entre guerres et paix. 1870-1995. Étude de biogéographie historique sur l'Arc meusien de l'Argonne à la Woëvre ", thèse de doctorat d'État, université de Lille, 1999.

Boulanger, P., « La forêt : des confins militaires à l'espace de guerre ", La Géographie, nº 1538, 2010, p. 25-31.

Boutefeu, B., « Le massif forestier, objet de mise en scène ", Revue Géographique de l'Est, vol. 49, $\mathrm{n}^{\text {os }}$ 2-3, 2009, mis en ligne en octobre 2010, URL : URL : http://journals.openedition.org/rge/1882

Buridant, J., « La forêt mutilée. La reconstitution forestière dans l'Aisne après la Grande Guerre ", Graines d'histoire, la mémoire de l'Aisne, $\mathrm{n}^{\circ} 17$, janvier 2003, p. 13-24.

Chiffre, E., Mathis, D., Michel, A., Müller, A., « Retracer le paysage : la ligne Maginot, de sa réalité matérielle à ses constructions imaginaire », dans Frangne, P.-H., Limido, P., Le Du, L., Regnauld, H., Des lignes et des paysages. Du sillon à la skyline, Rennes, Presses universitaires de Rennes, 2020, p. 181-206 (à paraître).

Chiffre, E. et Mathis, D., « De la mise en défense au déclassement d'un territoire : vers une réappropriation de l'empreinte militaire dans la région de Dijon-Langres ", Revue de Géographie historique, $\mathrm{n}^{\text {os }}$ 10-11, 2017, URL : http://rgh.univ-lorraine.fr/articles/view/94/ De_la_mise_en_defense_au_de...

Clément, G., Manifeste du tiers paysage, Paris, Éditions Jean-Michel Place, 2005, 69 p.

Dupré, L., « Conserver ensemble patrimoines naturel et culturel », Techniques \& Culture, 50, 2008, p. 84-101, mis en ligne en décembre 2010, URL : http://journals.openedition.org/tc/3943; DOI : https://doi.org/10.4000/tc.3943

Dupré, L., « Des friches : le désordre social de la nature », Terrain, $n^{\circ} 44,2005$, p. 125-136, mis en ligne en mars 2009, URL : http://journals.openedition.org/terrain/2488 ; DOI : 10.4000/terrain. 2488

Durup de Baleine, A., « Paysages hérités de la fortification "Séré de Rivières" au sein des Côtes lorraines ", Revue Géographique de l'Est, vol. 38, nos 1-2, 1998, p. 57-84, URL : https:// www.persee.fr/doc/rgest_0035-3213_1998_num_38_1_2483.

Ginet, P., « Les restructurations militaires en Lorraine : enjeux d'aménagement à différentes échelles ", Revue Géographique de l'Est, vol. 51, $\mathrm{n}^{\text {os }}$ 1-2, mis en ligne en décembre 2011, URL : http:// journals.openedition.org/rge/3242.

Husson, J.-P., et Rochel, X., « Avant-propos. Le massif forestier, espace géographique et territoire ", Revue Géographique de l'Est, vol. 49, ${ }^{\text {os }} 2-3,2009$, mis en ligne en octobre 2010, URL : http://journals.openedition.org/rge/1914

Husson, J.-P., " L'aménagement des forêts domaniales de Front de Haye et des Hauts de Mad », dans Amat, J.-P. et Corvol, A. (dir.), Forêt et Guerre, Paris, L'Harmattan, 1994, p. 197-213.

Labrue, C., «Le massif, ses marges et la préoccupation d'enfermement de l'habitat. Exemple des Vosges du Nord ", Revue Géographique de l'Est, vol. 49, $\mathrm{n}^{\text {os }} 2-3,2009$, mis en ligne en novembre 2010, URL : http://journals.openedition.org/rge/2007

Mathis, D., Chiffre, E. et Weimerskirch, G., « Les hydrosystèmes défensifs : des paysages militaires atypiques de la Ligne Maginot », Revue de Géographie historique, nos 10-11, mai 2017, URL : http:// 
rgh.univ-lorraine.fr/articles/view/92/

Les_hydrosystemes_defensifs_des_paysages_militaires_atypiques_de_la_Ligne_Maginot.

Mathis, D., « Les hydrosystèmes militaires défensifs de Basse-Alsace (xVII ${ }^{\mathrm{e}}$-XVIII ${ }^{\mathrm{e}}$ siècles) ", Revue de Géographie historique, n 8, mai 2016, URL : http://rgh.univ-lorraine.fr/articles/view/70/ Les_hydrosystemes_militaires_defensifs_de_Basse_Alsace_XVIIe_XVIIIe_siecles

Mathis, A. et Mathis, D., « Démilitarisation et reconversion de l'héritage militaire. L'exemple de Metz métropole ", Projets de paysage, $\mathrm{n}^{\circ} 11$, mis en ligne en janvier 2015, URL : https:// www.projetsdepaysage.fr/demilitarisation_et_reconversion_de_l_heritage_militaire

Mathis, D., « "Quand l'armée s'en va”. Approche géohistorique des restructurations des espaces militarisés : l'exemple du sud mosellan ", Revue Géographique de l'Est, vol. 51, n ${ }^{\text {os }} 1-2$, mis en ligne en décembre 2011, URL : http://journals.openedition.org/rge/3249

Matos-Machado, R. de, Amat, J.-P., Arnaud-Fassetta, G., Bétard, F., « Potentialités de l'outil LiDAR pour cartographier les vestiges de la Grande Guerre en milieu intra-forestier (bois des Caures, forêt domaniale de Verdun) », Échogéo, n 38, EchoGéo, mis en ligne en décembre 2016, URL : http://journals.openedition.org/echogeo/14791; DOI : 10.4000/echogeo.14791

Myard, J., « L'accord du 10 mai 1984 sur le Mundat », Annuaire français de droit international, vol. 31, 1985, p. 884-892, URL : https://www.persee.fr/doc/afdi_0066-3085_1985_num_31_1_2698.

Niederlander, T., "Relevé et chroniques des implantations militaires du territoire de Bitche », rapport de stage dans le cadre de l'OHM Pays de Bitche, M1 GAED, Nancy, 2019, 92 p.

Pardela, Ł., Wilkaniec, A., Środulska-Wielgus, J., Wielgus, K., 2018, « The importance of postfortress green areas for the urban green space in Cracow, Poznań and Wrocław ", Nauka Przyroda Technologie, vol. 12, $\mathrm{n}^{\circ}$ 2, p. 153-162, http://dx.doi.org/10.17306/J.NPT.00233

Puyot, J.-Y., « Les conséquences de la Première Guerre mondiale pour les forêts et les forestiers français ", Revue Forestière Française, $n^{\circ}$ 6, 2004, p. 103-132.

Schenk, W., 2013, « De l'espace résiduel aux paysages culturels : les nouvelles conceptions de la ruralité et de ses ressources dans les politiques territoriales en Allemagne », dans Lacquement, G., Born, K. M., Hirschhausen, B. von (dir.), Réinventer les campagnes en Allemagne. Paysage, patrimoine et développement rural, Lyon, ENS Éditions, 2013, URL : https:// books.openedition.org/enseditions/4583 ?lang $=\mathrm{fr}$

Sgard, A., « Entre rétrospective et prospective. Comment reconstruire le projet de territoire. ", EspacesTemps.net, mis en ligne en septembre 2008, URL : https://www.espacestemps.net/articles/ entre-retrospective-et-prospective/

Steinbach, F., Husson, J.-P., « Palimpsestes et héritages des polémopaysages dans les massifs du Saillant de Saint-Mihiel », dans Dupouey, J.-L., Dardignac, C. et Georges-Leroy, M., La Mémoire des forêts, actes du colloque " Forêt, archéologie et environnement ", 14-16 décembre 2004, Office national des forêts/Institut national de la recherche agronomique/Direction régionale des affaires culturelles de Lorraine, 2007, p. 285-294.

Taborelli, P., Devos, A., Laratte, S., Brenot, J., Bollot, N., Cancès, B., et Desfossés, Y., « Apport des plans directeurs et de l'outil LiDAR aéroporté pour la caractérisation des impacts morphologiques de la Grande Guerre : exemple de la cote 108 (Berry-au-Bac, France) », Géomorphologie. Relief, processus, environnement, vol. 23, $\mathrm{n}^{\circ}$ 2, mis en ligne en juin 2017, URL : http://journals.openedition.org/geomorphologie/11711; DOI : 10. 4000/geomorphologie.11711 


\section{NOTES}

1. Par hypermilitarisation, il faut comprendre la mise en œuvre d'un territoire de défense ou d'une métropole militaire concentrant les effectifs, les états-majors, les infrastructures logistiques et transformant une ville de garnison en agglomération avec la périphérisation des grands casernements (Mathis D et A., 2015).

2. Les polémopaysages regroupent les paysages de guerre, c'est-à-dire les lieux dévastés (sols, forêts, villages détruits) qui gardent d'importantes traces fossilisées et des séquelles environnementales. Dans cette étude nous différencierons les territoires forestiers militarisés des territoires forestiers marqués par la guerre, même si cette scission reste plus théorique que réelle.

3. Dans le cadre du dossier "Forêt d'Exception ", l'inventaire des vestiges de la ligne Maginot en forêt indivise de Haguenau a été réalisé entre mars et juillet 2018 par Paul Anglay, étudiant stagiaire en BTS gestion forestière, sous l'encadrement de Blaise Poudras, technicien forestier territorial à l'ONF et de Noémie Renaud-Goud, cheffe de projet et depuis secrétaire générale d'une agence ONF. Nous remercions Mme Renaud-Goud de nous avoir associés à cette démarche d'inventaire et MM. Blaise Poudras et Jacky Bock de nous avoir accompagnés sur le terrain et fait découvrir les éléments de la ligne sous forêt.

4. Ce travail a bénéficié d'une aide de l'État gérée par l'Agence nationale de la recherche au titre du Labex DRIIHM, programme «Investissements d'avenir» portant la référence ANR-11LABX-0010.

5. L'Obermundat se trouve au nord-ouest de Wissembourg et fait partie de la forêt du Palatinat. En 1815, la totalité de la forêt de l'obermundat, ainsi qu'une partie de la ville de Wissembourg furent cédées à la Bavière.

6. L'Untermundat (679 ha) a la particularité d'être la seule forêt domaniale française située à l'étranger. Le traité de Paris, promulgué en 1815, est source d'un litige qui ne trouvera son règlement qu'après un premier accord intervenu en 1984 (Myard, 1985). Les droits de la France ont été confirmés par un contrat établi à Nancy le 28 août 1990 entre les représentants de la République fédérale d'Allemagne, du Land de Rhénanie-Palatinat et de la République française. Le décret n 94-524 du 21 juin 1994 publié au JORF le 28 juin 1994.

7. Dans le cadre du projet de labellisation de la forêt indivise de Haguenau, un inventaire de terrain a été réalisé par l'Office national des forêts (ONF) afin d'évaluer l'importance de l'empreinte de la militarisation.

8. Schaller, F., Office de génie écologique (OGE), 420030070, vallée de la Lauter de Wissembourg à Scheibenhard. - INPN, SPN-MNHN Paris, 8P, URL: https://inpn.mnhn.fr/zone/znieff/ 420030070.pdf

9. Schaller, F., Office de génie écologique (OGE), op. cit, Natura 2000. Formulaire standard de données, pour les zones de protection spéciale (ZPS), les propositions de sites d'importance communautaire (pSIC), les sites d'importance communautaire (SIC) et les zones spéciales de conservation (ZSC), FR4201796 - La Lauter, https://inpn.mnhn.fr/docs/natura2000/fsdpdf/ FR4201796.pdf

10. Fédération allemande pour l'environnement et la protection de la nature, association non gouvernementale. 


\section{RÉSUMÉS}

Les territoires et paysages frontaliers de la région Grand-Est ont été fortement marqués par les infrastructures liées à la militarisation. Ils sont aujourd'hui frappés par un important processus de démilitarisation et d'effacement de cet héritage lié à la défense du territoire. Les massifs forestiers occupent une place particulière dans la préservation de l'empreinte militaire. C'est au cœur de ces espaces que les traces de la militarisation ont été les mieux préservées et fossilisées, à l'image des vestiges de la ligne Maginot ou de la ligne de fortifications de la Lauter. Cependant la gestion territoriale de ces massifs forestiers frontaliers du Pays de Bitche dans les Vosges du Nord, de Haguenau et du Bienwald-Mundat permet de souligner des approches et des trajectoires paysagères différentes de leur empreinte militaire.

The territories and landscapes in the border areas of the Grand-Est region have been strongly marked by military infrastructures. These areas are currently undergoing a major process of demilitarisation resulting in the disappearance of this national defence heritage. The forest massifs occupy a special place in the preservation of the military footprint. It is in the heart of these areas that traces of militarisation such as the remains of the Maginot line or the Lauter fortifications have been most effectively preserved within the landscape. However, the territorial management of these forest massifs on the borders of the Pays de Bitche in the Vosges du Nord and of Haguenau and Bienwald-Mundat reflects landscape approaches and trajectories which contrast with their military footprint.

\section{INDEX}

Mots-clés : trajectoires, territoire de défense, paysage militarisé, Forêt d'Exception, paysage culturel

Keywords : trajectories, defended territory, militarised landscape, special forest, cultural landscape

\section{AUTEURS}

\section{DENIS MATHIS}

Denis Mathis est maître de conférences, université de Lorraine, LOTERR. Ses domaines de recherche : géohistoire, dynamiques paysagères, territoires d'eau, territoires militarisés, démilitarisation, transitions et reconversions, tourisme de mémoire.

denis.mathis[at]univ-lorraine[dot]fr

\section{EMMANUEL CHIFFRE}

Emmanuel Chiffre est maître de conférences, université de Lorraine, LOTERR. Ses domaines de recherche : géographie militaire, frontières, villes de garnison, fortifications, démilitarisation et transitions des territoires de défense, reconversions, patrimonialisation, tourisme de mémoire. Emmanuel.chiffre[at]univ-lorraine[dot]fr

\section{CHRISTELLE BALOUZAT-LOUBET}

Christelle Balouzat-Loubet est maître de conférences en histoire médiévale, université de Lorraine, CRUHL. Ses domaines de recherche sont tournés vers l'édition numérique de 
documents médiévaux et l'histoire des principautés médiévales, particulièrement sur l'espace forestier lorrain en tant que miroir de l'affirmation du pouvoir princier entre les XIII ${ }^{\mathrm{e}}$ et $\mathrm{X}^{\mathrm{e}}$ siècles. Christelle.Loubet[at]univ-Lorraine[dot]fr

\section{TANGUY NIEDERLANDER}

Tanguy Niederlander est étudiant en master de géographie. Stagiaire à l'OHM Pays de Bitche, AAP 2019, il a travaillé sur l'appel à projets « L'empreinte militaire au Pays de Bitche du Moyen Âge à nos jours ».

tanguy.niederlander1[at]etu.univ-lorraine[dot]fr

\section{ANNE MATHIS}

Anne Mathis est enseignante et chercheuse associée, université de Lorraine, LOTERR. Ses domaines de recherche : écoquartier, reconversion urbaine, valorisation, patrimonialisation, réhabilitation de sites militaires.

anne.mathis[at]ac-nancy-metz[dot]fr 